University of Texas Rio Grande Valley

ScholarWorks @ UTRGV

Physics and Astronomy Faculty Publications and Presentations

College of Sciences

$5-2020$

\title{
Fingerprints of Binary Black Hole Formation Channels Encoded in the Mass and Spin of Merger Remnants
}

\author{
Manuel Arca Sedda \\ Michaela Mapelli \\ Mario Spera \\ Matthew Benacquista \\ The University of Texas Rio Grande Valley \\ Nicola Giacobbo
}

Follow this and additional works at: https://scholarworks.utrgv.edu/pa_fac

Part of the Astrophysics and Astronomy Commons, and the Physics Commons

\section{Recommended Citation}

Sedda, Manuel Arca, et al. "Fingerprints of Binary Black Hole Formation Channels Encoded in the Mass and Spin of Merger Remnants." The Astrophysical Journal, vol. 894, no. 2, American Astronomical Society, May 2020, p. 133, doi:10.3847/1538-4357/ab88b2.

This Article is brought to you for free and open access by the College of Sciences at ScholarWorks @ UTRGV. It has been accepted for inclusion in Physics and Astronomy Faculty Publications and Presentations by an authorized administrator of ScholarWorks @ UTRGV. For more information, please contact justin.white@utrgv.edu, william.flores01@utrgv.edu. 


\title{
Fingerprints of Binary Black Hole Formation Channels Encoded in the Mass and Spin of Merger Remnants
}

\author{
Manuel Arca Sedda ${ }^{1}$ (D), Michela Mapelli ${ }^{2,3,4}$ (D), Mario Spera ${ }^{2,3,5,6}$ (D), Matthew Benacquista ${ }^{7,8}$, and Nicola Giacobbo ${ }^{2,3,4}$ (iD \\ ${ }^{1}$ Zentrum für Astronomie der Universität Heidelberg, Astronomisches Rechen-Institut, Mönchhofstrasse 12-14, Heidelberg, D-69120, Germany \\ m.arcasedda@gmail.com \\ ${ }^{2}$ Physics and Astronomy Department Galileo Galilei, University of Padova, Vicolo dell'Osservatorio 3, I-35122, Padova, Italy \\ ${ }^{3}$ INFN-Padova, Via Marzolo 8, I-35131 Padova, Italy \\ ${ }_{5}^{4}$ INAF-Osservatorio Astronomico di Padova, Vicolo dell'Osservatorio 5, I-35122, Padova, Italy \\ ${ }^{5}$ Department of Physics and Astronomy, Northwestern University, Evanston, IL 60208, USA \\ ${ }^{6}$ Center for Interdisciplinary Exploration and Research in Astrophysics (CIERA), Northwestern University, Evanston, IL 60208, USA \\ ${ }^{7}$ Center for Gravitational Wave Astronomy, University of Texas Rio Grande Valley, One University Blvd, Brownsville, TX 78520, USA \\ ${ }^{8}$ Division of Astronomy, National Science Foundation, 2415 Eisenhower Ave, Alexandria, VA 22314, USA \\ Received 2020 January 6; revised 2020 April 9; accepted 2020 April 9; published 2020 May 15
}

\begin{abstract}
Binary black holes (BBHs) are thought to form in different environments, including the galactic field and (globular, nuclear, young, and open) star clusters. Here, we propose a method to estimate the fingerprints of the main $\mathrm{BBH}$ formation channels associated with these different environments. We show that the metallicity distribution of galaxies in the local universe along with the relative amount of mergers forming in the field or in star clusters determine the main properties of the $\mathrm{BBH}$ population. Our fiducial model predicts that the heaviest merger to date, GW170729, originated from a progenitor that underwent 2-3 merger events in a dense star cluster, possibly a galactic nucleus. The model predicts that at least one merger remnant out of a hundred BBH mergers in the local universe has mass $90<M_{\mathrm{rem}} / M_{\odot} \leqslant 110$, and one in a thousand can reach a mass as large as $M_{\text {rem }} \gtrsim 250 M_{\odot}$. Such massive black holes would bridge the gap between stellar-mass and intermediate-mass black holes. The relative number of low- and high-mass BBHs can help us unravel the fingerprints of different formation channels. Based on the assumptions of our model, we expect that isolated binaries are the main channel of BBH merger formation if $\sim 70 \%$ of the whole $\mathrm{BBH}$ population has remnants with masses $<50 M_{\odot}$, whereas $\gtrsim 6 \%$ of remnants having masses $>75 M_{\odot}$ points to a significant subpopulation of dynamically formed BBH binaries.
\end{abstract}

Unified Astronomy Thesaurus concepts: Galaxy nuclei (609); Star clusters (1567); Astrophysical black holes (98); Gravitational waves (678); Stellar evolution (1599)

\section{Introduction}

The direct detection of gravitational waves (GWs) by the LIGO_-Virgo collaboration (LVC; Abbott et al. 2016a, 2016b, 2016c, 2017a, 2017b, 2017c, 2019a) has marked the dawn of GW astronomy.

During the first two observational runs (Abbott et al. 2019a), the LVC detected ten binary black hole $(\mathrm{BBH})$ mergers (Abbott et al. 2016a, 2016b, 2016c, 2017a, 2017b, 2017c, 2019a) and one double neutron star merger (Abbott et al. 2017d). The black holes (BHs) detected thus far are consistent with a powerlaw mass distribution with index $1.3_{-1.7}^{+1.4}$ (at 90\% confidence level) and no more than $\sim 1 \%$ BHs with mass $>45 M_{\odot}$ (Abbott et al. 2019b).

One of the intriguing puzzles related to LVC detections is the large mass of the observed mergers. Indeed, 8 out of 10 detected BBHs have components with masses above $20 M_{\odot}$. This seeming overabundance of heavy stellar BHs contrasts with the $22 \mathrm{BHs}$ observed in X-ray binaries that have masses in the range 1.6-18M $\odot$ (Remillard \& McClintock 2006; Casares et al. 2017).

This difference between the mass range of $\mathrm{LVC} \mathrm{BHs}$ and BHs in X-ray binaries might be ascribed to the detector's sensitivity, which is much higher for larger BH masses (see, for instance, Fishbach et al. 2017), to other observational biases (e.g., X-ray binaries for which we have a dynamical mass measurement are within few Mpc in a predominantly metal-rich environment), to a predominantly different formation channel
(Perna et al. 2019), or to gravitational lensing (Broadhurst et al. 2018). One of the critical parameters affecting the natal mass of BHs is the metallicity of their progenitors, $Z$, as metal-poor stars are expected to produce heavier BHs (Mapelli et al. 2009, 2010; Belczynski et al. 2010; Mapelli \& Bressan 2013; Spera et al. 2015) and to have a higher merger efficiency than metal-rich stars (Dominik et al. 2013; Askar et al. 2017; Giacobbo et al. 2018). Merging BBHs form either from isolated binary stellar evolution in galactic fields (Tutukov \& Yungelson 1973; Portegies Zwart \& McMillan 2000; Belczynski et al. 2002, 2010, 2016a; Hurley et al. 2002; Mapelli \& Bressan 2013; Marchant et al. 2016; Giacobbo et al. 2018; Arca Sedda \& Benacquista 2019; Spera et al. 2019, and references therein), or through dynamical interactions in dense young massive clusters (Portegies Zwart \& McMillan 2000; Banerjee et al. 2010; Ziosi et al. 2014; Mapelli 2016; Banerjee 2017, 2018; di Carlo et al. 2019a; Rastello et al. 2019), globular clusters (Sigurdsson \& Phinney 1993; Lee 1995; Miller \& Hamilton 2002; Wen 2003; O’Leary et al. 2009; Downing et al. 2010; Rodriguez et al. 2015; Antonini et al. 2016; Rodriguez et al. 2016; Askar et al. 2017; Arca Sedda et al. 2018; Hong et al. 2018; Rodriguez \& Loeb 2018; Samsing 2018; Zevin et al. 2019), or nuclear clusters and galactic nuclei (Miller \& Lauburg 2009; Antonini \& Rasio 2016; Bartos et al. 2017; O'Leary et al. 2016; Stephan et al. 2016; VanLandingham et al. 2016; Antonini et al. 2019; Arca Sedda \& Gualandris 2018; Fragione et al. 2019; 
Hoang et al. 2018; Arca Sedda \& Capuzzo-Dolcetta 2019; Arca Sedda 2020).

The main properties of merging BBHs-component masses, semimajor axis, and eccentricity-depend primarily on their birth site. Mergers taking place in galactic fields are usually characterized by low eccentricities unless they are part of a hierarchical triple (Antonini et al. 2017). In star clusters, instead, the zoology of BBH mergers is quite vast. Dynamical scatterings can drive the shrinkage of a $\mathrm{BBH}$ down to a point where GWs dominate the evolution (Arca Sedda et al. 2018; Rodriguez et al. 2018; Samsing 2018; Zevin et al. 2019), or can trigger the formation of triples that can efficiently affect the BBH end phase for both stable (Antonini et al. 2017; Rastello et al. 2019) or unstable systems (Arca Sedda et al. 2018).

Dense stellar systems, like globular or nuclear clusters, can potentially retain merger products and favor multiple mergers, thus allowing BH mass buildup (Fishbach et al. 2017; Antonini et al. 2019; Gerosa et al. 2018; Qin et al. 2018; Arca Sedda \& Benacquista 2019; Doctor et al. 2020; Rodriguez et al. 2019). These second generation $\mathrm{BHs}$ can significantly affect the $\mathrm{BH}$ mass spectrum. In galactic nuclei, $\mathrm{BBH}$ evolution and coalescence is even more complex due to the possible presence of a quiescent SMBH (Antonini \& Perets 2012; Arca Sedda \& Gualandris 2018; Hoang et al. 2018; Arca Sedda \& CapuzzoDolcetta 2019; Fernández \& Kobayashi 2019; Hoang et al. 2019; Rasskazov \& Kocsis 2019) or an AGN in the galactic center (Bartos et al. 2017; Yang et al. 2019).

Placing constraints on the population of merger products is also important to improve our knowledge of $\mathrm{BH}$ formation. For instance, chirp masses can be used to constrain the global BH natal kick distribution (Zevin et al. 2017; Barrett et al. 2018), while their spin distribution can carry information on BH natal spins and BBH spin alignment in isolated (Gerosa et al. 2018) and dynamical environments (Morawski et al. 2018). As LIGO and Virgo reach full sensitivity and the number of detections increases, it will be possible to determine the most likely BH spin amplitude and the BBH spin orientation (Farr et al. 2017; Stevenson et al. 2017; Talbot \& Thrane 2017; Arca Sedda \& Benacquista 2019; Bouffanais et al. 2019; Bavera et al. 2020). Dissecting the formation history of BBHs from GW observations is a multifaceted problem that requires simultaneous accounting for single and binary stellar evolution, stellar dynamics, general relativity, and cosmology. Addressing this problem by means of direct $N$-body simulations combined with population-synthesis simulations is a computational challenge (see, e.g., Wang et al. 2016; Banerjee 2017; di Carlo et al. 2019a; Rastello et al. 2019). If we want to probe a large portion of the parameter space, we need a much faster approach than full $N$-body simulations. Recently, Arca Sedda \& Benacquista (2019) proposed a way to take into account these different aspects with a fast and self-consistent approach. Following a similar technique, in this paper we provide an astrophysical framework to characterize the formation channels of BBHs.

We combine state-of-the-art stellar evolution recipes, theoretical models for BBH merger processes, observational constraints on the local universe metallicity distribution, and numerical relativity fitting formulae to calculate post-merger BHs' final mass and spin. We explore how theoretical uncertainties can affect the results, and discuss what we can learn from potential differences between observations and our model.

The paper is organized as follows. In Section 2, we discuss the method and the underlying assumptions beneath our fiducial model. Section 3 presents the main results of our fiducial model, providing a comparison to the known population of GW sources (Section 3.2), showing how different formation channels impact the percentage of massive mergers in $\mathrm{BBH}$ populations (Section 3.3), and discussing how such a model can be used to constrain the formation pathway of massive BBH mergers like GW170729 (Section 3.4). Section 4 is devoted to investigating the impact of theoretical uncertainties on our results. In Section 5, we present the conclusions of this work.

\section{Method}

Tracking the evolutionary path of merging BBHs requires taking into account several parameters simultaneously: the metallicity distribution of galaxies and star clusters in the local universe; the possibility that GW observations are biased toward heavy mergers; and the probability for a merger to take place in metal-poor or metal-rich environments, in the field or in a star cluster.

Our multistep procedure can be outlined as follows. For each $\mathrm{BBH}$ that coalesces, we:

1. select its birth-place metallicity;

2. select the BBH formation channel assuming different probability thresholds for isolated and dynamical channels;

3. use single/binary stellar evolution to calculate the natal mass of the components, taking into account an observational selection function to select the BBH primary mass;

4. calculate the natal spin amplitude of the components;

5. calculate the orientation of the spins according to a given distribution;

6. calculate the merged $\mathrm{BH}$ final mass and spin via numerical relativity fitting formulae.

The procedure is sketched in Figure 1.

Table 1 summarizes the features of our fiducial model and of the other models we consider to estimate the impact of our assumptions on the final results. For each model, we create a sample of $10^{5} \mathrm{BBH}$ mergers.

The range of assumptions featured by our fiducial model is detailed in the following subsections.

\subsection{Metallicity Distribution}

In order to obtain a reliable distribution for the metallicity of BBH host galaxies, we use the analysis performed by Gallazzi et al. (2005), based on 44254 galaxies drawn from the Sloan Digital Sky Survey Data Release Two (SDSS DR2). Note that the galaxy sample considered here spans the redshift range $0.005<z \leqslant 0.22$. Thus, it provides a reliable representation of the volume scanned by the LVC during the $\mathrm{O} 1$ (and part of the O2) runs. ${ }^{9}$

As shown in Figure 2, the metallicity distribution shows a clear peak toward solar values, while the population of metalpoor galaxies $\left(Z<0.1 Z_{\odot}\right)$ accounts for less than a few percent of all the galaxies in the sample.

The preponderance of metal-rich galaxies might have a major impact on the mass of BBH mergers, as metal-rich stars are expected to produce lower-mass BHs (Mapelli et al. 2009; Belczynski et al. 2010; Mapelli et al. 2010; Mapelli \& Bressan 2013; Spera et al. 2015; Belczynski et al. 2016b; Giacobbo et al. 2018) and to lead to smaller merger efficiency

\footnotetext{
9 The instrumental horizon of LIGO and Virgo was $\sim 1.3$ Gpc (Martynov et al. 2016) (redshift $z \simeq 0.25$ ) during $\mathrm{O} 1$, and will grow up to $4 \mathrm{Gpc}(z \sim 1)$ at design sensitivity (Abbott et al. 2019a)
} 


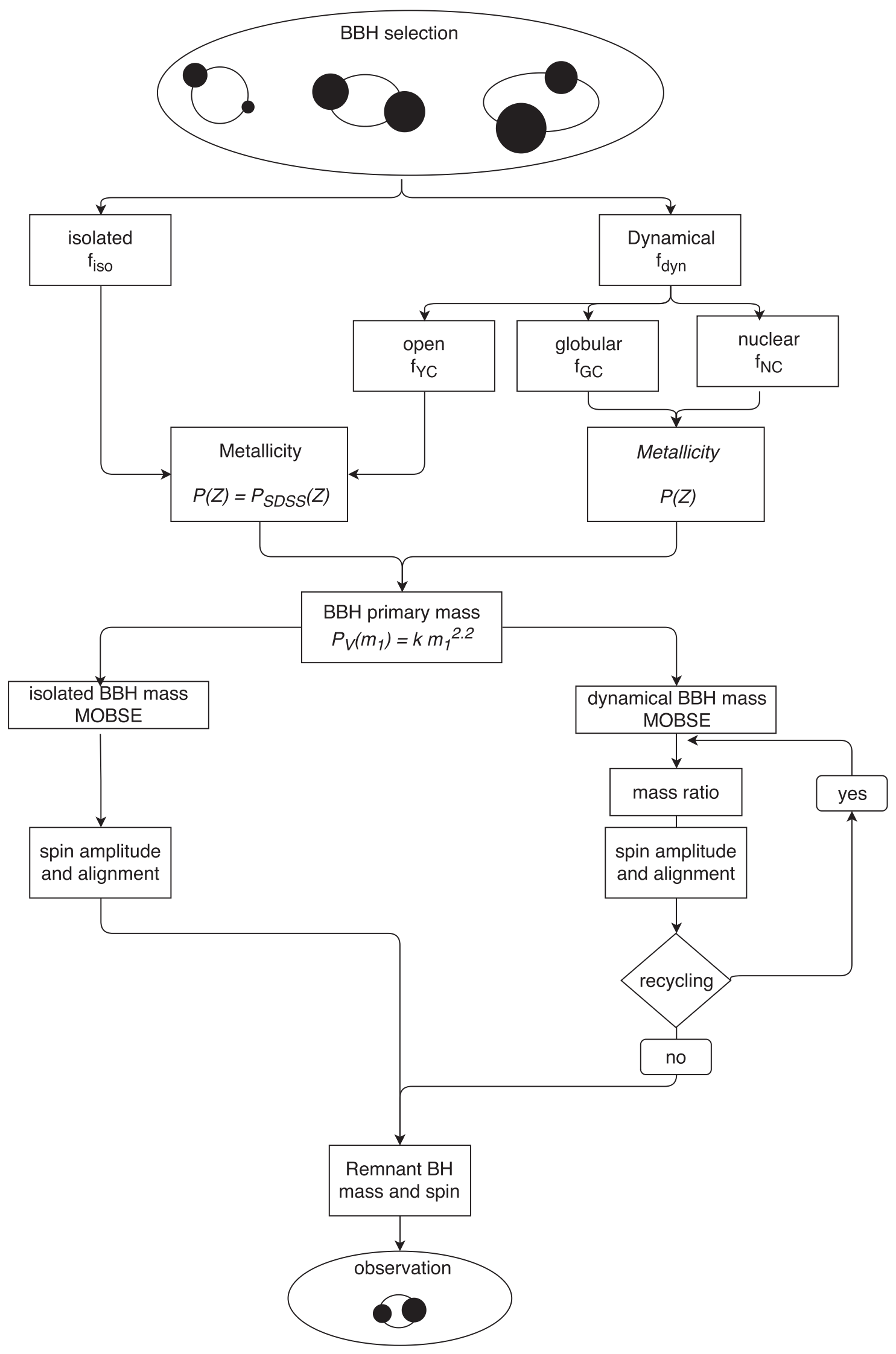

Figure 1. Sketch of the procedure adopted to create the BBH catalog. 
Table 1

Parameters of the Models Investigated

\begin{tabular}{|c|c|c|c|c|c|c|c|c|c|c|c|c|c|c|}
\hline \multirow[t]{3}{*}{ ID } & \multicolumn{2}{|c|}{$\begin{array}{c}\text { Formation } \\
\text { Channel }\end{array}$} & \multicolumn{6}{|c|}{ Dynamical Channel } & \multicolumn{3}{|c|}{ Metallicity } & \multirow{3}{*}{$\frac{\text { OBS }}{\alpha_{m_{1}}}$} & \multicolumn{2}{|c|}{ Spins } \\
\hline & \multirow[t]{2}{*}{$f_{\text {iso }}$} & \multirow[t]{2}{*}{$f_{\mathrm{dyn}}$} & \multicolumn{2}{|c|}{$f_{\mathrm{GC}}$} & \multicolumn{2}{|c|}{$f_{\mathrm{NC}}$} & \multicolumn{2}{|c|}{$f_{\mathrm{YC}}$} & \multicolumn{2}{|c|}{$P(Z)$} & \multirow[t]{2}{*}{$f(Z)$} & & \multirow[t]{2}{*}{$P\left(a_{1}\right)$} & \multirow[t]{2}{*}{$n_{\theta}$} \\
\hline & & & $q_{\min }$ & $\begin{array}{c}v_{\max } \\
\left(\mathrm{km} \mathrm{s}^{-1}\right)\end{array}$ & $q_{\min }$ & $\begin{array}{c}v_{\max } \\
\left(\mathrm{km} \mathrm{s}^{-1}\right)\end{array}$ & $q_{\min }$ & $\begin{array}{c}v_{\max } \\
\left(\mathrm{km} \mathrm{s}^{-1}\right)\end{array}$ & iso $+y c$ & $\mathrm{gc}+\mathrm{nc}$ & & & & \\
\hline \multicolumn{15}{|c|}{ Fiducial model } \\
\hline \multirow[t]{2}{*}{1} & 0.67 & 0.33 & \multicolumn{2}{|c|}{0.6} & \multicolumn{2}{|c|}{0.2} & \multicolumn{2}{|c|}{0.2} & \multirow[t]{2}{*}{ SDSS } & \multirow[t]{2}{*}{ LOG } & \multirow[t]{2}{*}{$Z^{\beta}$} & \multirow[t]{2}{*}{2.2} & \multirow[t]{2}{*}{ uniform } & 0 \\
\hline & & & 0.2 & 15 & 0.2 & 100 & 0 & 3 & & & & & & \\
\hline & & & & & & Choice & metal & distribut & & & & & & \\
\hline $2 \mathrm{a}$ & 0.67 & 0.33 & & 6 & & 2 & & & SDSS & SDSS & 1 & 2.2 & uniform & 0 \\
\hline & & & 0.2 & 15 & 0.2 & 100 & 0 & 3 & & & & & & \\
\hline $2 b$ & 0.67 & 0.33 & & 6 & & 2 & & & SDSS & LOG & 1 & 2.2 & uniform & 0 \\
\hline & & & 0.2 & 15 & 0.2 & 100 & 0 & 3 & & & & & & \\
\hline $2 \mathrm{c}$ & 0.67 & 0.33 & & 6 & & 2 & & & LOG & LOG & 1 & 2.2 & uniform & 0 \\
\hline & & & 0.2 & 15 & 0.2 & 100 & 0 & 3 & & & & & & \\
\hline & & & & & & Cho & of nat & vironmen & & & & & & \\
\hline $3 \mathrm{a}$ & 1 & 0 & & . & & - & & & SDSS & $\cdots$ & $Z^{\beta}$ & 2.2 & uniform & 0 \\
\hline & & & $\cdots$ & $\cdots$ & $\cdots$ & $\cdots$ & $\cdots$ & $\cdots$ & & & & & & \\
\hline $3 b$ & 1 & 0 & & . & & . & & & SDSS /30 & $\cdots$ & $Z^{\beta}$ & 2.2 & uniform & 0 \\
\hline & & & $\cdots$ & $\cdots$ & $\cdots$ & $\cdots$ & $\cdots$ & $\cdots$ & & & & & & \\
\hline $3 \mathrm{c}$ & 1 & 0 & & & & . & & & LOG & $\ldots$ & $Z^{\beta}$ & 2.2 & uniform & 0 \\
\hline & & & $\cdots$ & $\cdots$ & $\cdots$ & $\cdots$ & $\cdots$ & $\cdots$ & & & & & & \\
\hline $4 a$ & 0 & 1 & & 6 & & 2 & & & SDSS & LOG & $Z^{\beta}$ & 2.2 & uniform & $\ldots$ \\
\hline & & & 0.2 & 15 & 0.2 & 100 & 0 & 3 & & & & & & \\
\hline $4 b$ & 0 & 1 & & 0 & & 0 & & & $\cdots$ & LOG & $Z^{\beta}$ & 2.2 & uniform & $\cdots$ \\
\hline & & & 0.2 & 15 & $\cdots$ & $\cdots$ & $\cdots$ & $\cdots$ & & & & & & \\
\hline $4 c$ & 0 & 1 & & 0 & & 0 & & & SDSS & $\ldots$ & $Z^{\beta}$ & 2.2 & uniform & $\cdots$ \\
\hline & & & $\cdots$ & $\cdots$ & $\cdots$ & $\cdots$ & 0 & 3 & & & & & & \\
\hline $4 d$ & 0 & 1 & & 0 & & 0 & & & $\ldots$ & LOG & $Z^{\beta}$ & 2.2 & uniform & $\ldots$ \\
\hline & & & $\cdots$ & $\cdots$ & 0.2 & 100 & $\cdots$ & $\cdots$ & & & & & & \\
\hline $4 \mathrm{~d} \dagger$ & 0 & 1 & & 0 & & 0 & & & $\ldots$ & LOG & $Z^{\beta}$ & 2.2 & uniform & $\ldots$ \\
\hline & & & $\cdots$ & $\cdots$ & 0.2 & 100 & $\cdots$ & $\cdots$ & & & & & & \\
\hline $4 \mathrm{e}$ & 0 & 1 & & 0 & & 0 & & & $\ldots$ & LOG & $Z^{\beta}$ & 2.2 & uniform & $\ldots$ \\
\hline & & & $\cdots$ & $\cdots$ & 0.2 & 0 & $\cdots$ & $\cdots$ & & & & & & \\
\hline 5 & 0.50 & 0.50 & & 33 & & 33 & & & SDSS & LOG & $Z^{\beta}$ & 2.2 & uniform & 0 \\
\hline & & & 0.2 & 15 & 0.2 & 100 & 0 & 3 & & & & & & \\
\hline
\end{tabular}

Notes. Col. 1: model ID number. Cols. 2-3: fractional number of isolated or dynamical mergers, respectively. Cols. 4-6: fractional number of sources forming in globular, nuclear, and young clusters. Sub-rows indicate, for each cluster type, the minimum mass ratio allowed and maximum velocity ( $\left.v_{\max }\right)$ allowed for a merger to be retained an undergo a further merger. Cols. 7-8: metallicity distribution adopted, either the one inferred from observations (SDSS) or flat in logarithmic values (LOG), and the weighting function used to account for the dependence between metallicity and merger probability. Col. 9: Slope of the observational mass selection function. Col. 10: natal spin distribution. Col. 11: slope of the distribution function adopted to model spins alignment: isotropic $\left(n_{\theta}=0\right)$, mildly aligned $\left(n_{\theta}=2\right)$, or fully aligned $\left(n_{\theta} \rightarrow \infty\right)$ distribution. In model $4 \mathrm{~d}, \dagger$ we assume the same values of set $4 \mathrm{~d}$, but the maximum mass allowed for single $\mathrm{BHs}$ is set to $40 M_{\odot}$.

(Dominik et al. 2013, 2015; Belczynski et al. 2016b; Rodriguez et al. 2016; Askar et al. 2017; Giacobbo et al. 2018). However, there are at least two aspects that should be considered here. On the one hand, a galaxy can be characterized by an intrinsic metallicity spread of up to 0.3 dex (see, for instance, Pilyugin et al. 2014). On the other hand, star clusters do not necessarily feature their host galaxy metallicity. This is clearly seen in our Galaxy. Indeed, while open clusters trace the Milky Way metallicity gradient fairly well (Netopil et al. 2016), the metallicity of globular clusters is significantly lower than that of disk stars (Harris et al. 2014). The Milky Way nuclear cluster, which consists of stars with a large spread in age and metallicity (Do et al. 2015), is characterized by a complex star formation history, similar to its extra-galactic counterparts (Rossa et al. 2006).

On top of this, population synthesis and $N$-body simulations suggest that the number of mergers strongly decreases at metallicity $Z>10^{-3}$ for both isolated (see, for instance, 


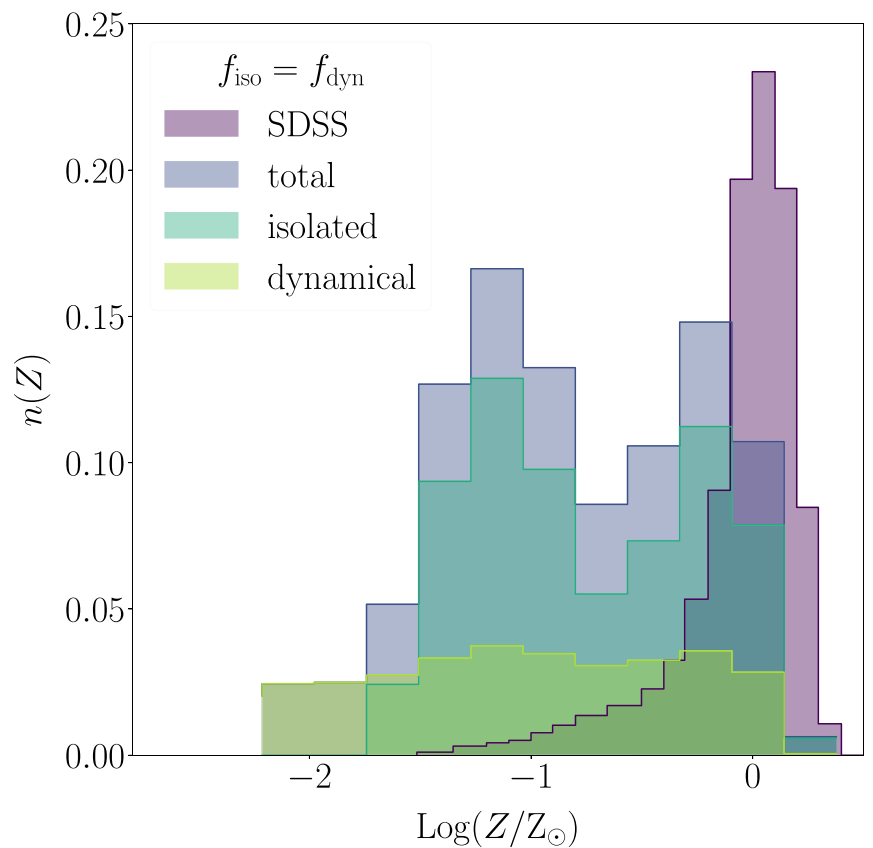

Figure 2. Metallicity distribution for isolated (dark green filled steps) and dynamical mergers (light green filled steps), overlaid on the metallicity distribution from the SDSS (purple filled steps). Total population of dynamical and isolated mergers is also shown (dark blue filled steps). Histogram is normalized to the total number of sources. We assume that the dynamical mergers are half of the total population, corresponding to model ID 5. We also assume that all dynamical environments (globular, young, and nuclear clusters) contribute equally, their fraction being $f_{\mathrm{GC}}=f_{\mathrm{YC}}=f_{\mathrm{NC}}$.

Giacobbo et al. 2018) and dynamical (Askar et al. 2017) BBH mergers.

In order to include all these features in our model, we create a two-layer procedure to select the $\mathrm{BBH}$ birth-site metallicity.

First, we assume that galaxies and open clusters are characterized by a metallicity distribution, $P(Z)$, equal to the one inferred from SDSS DR2 observations $P(Z) \equiv P_{\text {SDSS }}(Z)$. Note that this selection procedure allows us to naturally take into account the observed mass-metallicity relation. For globular and nuclear clusters, instead, we allow two possible choices: (i) same as for galaxies and open clusters; or (ii) logarithmically flat distribution, $P(\log Z)=$ const. Observations of globular clusters indicate a bimodal metallicity distribution whose properties, e.g., peak amplitudes, broadening, or limiting values, vary noticeably from one galaxy to another (see, e.g., Lamers et al. 2017), whereas nuclear clusters feature metallicities broadly distributed from subsolar to solar values (Rossa et al. 2006; Paudel et al. 2011; Neumayer et al. 2020). Our choice for the metallicity distribution of globular clusters and nuclear star clusters matches the main features of the complex metallicity distribution observed in globular and nuclear clusters in different environments, while keeping our model as simple as possible. Second, we weight the metallicity distribution with the probability for a merger to take place in an environment with a given $Z$. For simplicity, we assume that such probability has a power-law form, $f(Z) \propto Z^{\beta}$, with $\beta=-1.5$, to be consistent with isolated binaries' (Giacobbo et al. 2018) and star clusters' (Askar et al. 2017) results. The quantity $f(Z) P(Z)$ represents the probability for a merger to take place in galaxies at different metallicity. In all the models we consider, metallicity values are in the range $0.0002 \leqslant Z \leqslant 0.03$.

Figure 2 compares this quantity, assuming that the metallicitymerger dependence is either absent (and thus the probability to detect a BBH with progenitor metallicity $Z$ depends solely on the observed metallicity distribution) or is a power law. In the latter case, we also dissect the distribution into star clusters and galactic field, assuming that the population is equally divided between dynamical and isolated mergers. The plot shows how effective the contribution of metal-poor galaxies can be to the overall $\mathrm{BBH}$ merger population, if the $f(Z)$ dependence is taken into account. In our fiducial model, the metallicity selection for $\mathrm{BBH}$ progenitors is weighted with a power law $f(Z) \propto Z^{-1.5}$ and following the SDSS distribution $\left(P(Z)=P_{\text {SDSS }}\right)$ for isolated and open clusters $\mathrm{BBHs}$, or a logarithmically flat distribution $(P(\log Z)=$ const $)$ for globular and nuclear clusters BBHs.

We explore the effects of different choices in Section 4.1.

\subsection{Observational Selection Bias}

The actual size of the volume, $V$, visible to LIGO and Virgo depends in a nontrivial way on different parameters. More massive mergers emit GWs with higher strain amplitudes, and hence they are observable from greater distances. On the other hand, the GW frequency at merger is lower for higher-mass systems, leading the signal-to-noise ratio to be smaller, as the signal spends less time in the detector sensitivity band. The exact relation connecting $V$ and the merger properties also involves sky location, angle of inclination, and component spins. However, in the BBH mass range $^{10} 10<M_{\mathrm{BBH}} / M_{\odot}<100$, the mass dependence of the detection volume scales with a power law of the primary mass $m_{1}$, namely $V \propto m_{1}^{\delta}$ (Fishbach \& Holz 2017), with $\delta=2.2$. This relation is valid at fixed mass ratio and under the assumption that the spin does not affect the BBH detectability (Fishbach \& Holz 2017; Abbott et al. 2019b). Note that, at fixed $\mathrm{BBH}$ mass, lower mass ratios correspond to smaller volumes. The spin dependence can increase the volume up to $30 \%$, depending on the binary components' masses (Capano et al. 2016), although it is less trivial to show how spins affect the volume-binary total mass dependence. In our earlier paper, we explored how different choices for this relation affect the global population of observed BBH mergers (Arca Sedda \& Benacquista 2019).

Unless otherwise stated, throughout the paper we assume the power-law dependence $V \propto m_{1}^{2.2}$.

\subsection{Formation Channel Probability}

In this work, we consider either an isolated formation channel, namely the BBHs resulting from isolated evolution of a stellar binary, or a dynamical formation channel, according to which a $\mathrm{BBH}$ forms in star clusters from repeated scatterings among BHs originating via single stellar evolution.

The probability for a $\mathrm{BBH}$ merger to have originated via one mechanism or the other depends on our knowledge of the processes that regulate the $\mathrm{BBH}$ formation itself. A possible way to quantify such a probability is by comparing the theoretical merger rates, namely the number of events taking place per time unit and volume unit, obtained for both channels, and compare this to observational limits, namely $\Gamma=9.7-101 \mathrm{yr}^{-1} \mathrm{Gpc}^{-3}$ based on the 10 current detections (Abbott et al. 2019a, 2019b). The most recent calculations suggest the isolated channel's merger rate to be in the range $\Gamma \sim 10-250 \mathrm{yr}^{-1} \mathrm{Gpc}^{-3}$ (Dominik et al. 2013; Belczynski et al. 2016b; Eldridge et al. 2017; Mapelli et al. 2017; Kruckow et al. 2018; Mapelli \& Giacobbo 2018;

\footnotetext{
${ }^{10}$ In the following, we use $M_{\mathrm{BBH}}$ to refer to the sum of the $\mathrm{BBH}$ components mass, and $M_{\text {rem }}$ to refer to the mass of the merger remnant.
} 
Arca Sedda \& Benacquista 2019; Giacobbo \& Mapelli 2020; Neijssel et al. 2019; Spera et al. 2019). For the dynamical channel, instead, the merger rate depends on the type of hosting cluster, being $\Gamma \sim 5-50 \mathrm{yr}^{-1} \mathrm{Gpc}^{-3}$ for globular clusters (Rodriguez et al. 2016; Askar et al. 2017; Rodriguez et al. 2018; Arca Sedda \& Benacquista 2019), $\Gamma \sim 0.1-5 \mathrm{yr}^{-1} \mathrm{Gpc}^{-3}$ for open star clusters (Banerjee 2017; Kumamoto et al. 2019; Rastello et al. 2019), $\Gamma \sim 0.1-100$ for young star clusters (Ziosi et al. 2014; Mapelli 2016; di Carlo et al. 2019a, 2020), and $\Gamma \sim 0.5-10 \mathrm{yr}^{-1}$ $\mathrm{Gpc}^{-3}$ for nuclear clusters (Antonini \& Rasio 2016; VanLandingham et al. 2016; Arca Sedda \& Gualandris 2018; Hoang et al. 2018; Hong et al. 2018; Arca Sedda \& Capuzzo-Dolcetta 2019; Rasskazov \& Kocsis 2019).

Alternative theories for $\mathrm{BBH}$ formation, like primordial $\mathrm{BHs}$, lead to merger rates similar to those investigated here (see, for instance, Bird et al. 2016).

For clarity's sake, we build up our mock sample assuming that isolated mergers have a probability $f_{\text {iso, }}$, with a complementary probability $f_{\text {dyn }}=1-f_{\text {iso }}$ for dynamical mergers. According to this definition, in a sample of $N$ sources we will have, on average, $f_{\text {iso }} N$ isolated mergers and $f_{\text {dyn }} N$ dynamical mergers. For each $\mathrm{BBH}$, we draw a number $n$ between 0 and 1, assuming a flat distribution. In the case $n<f_{\text {iso }}$, the $\mathrm{BBH}$ is assumed to be isolated; otherwise, it is dynamical. Thus, the actual number of BBHs associated with a channel or the other will be affected by the statistical fluctuations inherent in the selection process.

In the fiducial model, we assume $f_{\text {iso }}=3 f_{\text {dyn }}$. Moreover, a further layer of complexity needs to be added to properly model dynamical mergers, for three main reasons. The first is connected to the evidence that different cluster types are characterized by different merger rates, although the amplitude of such difference is highly uncertain. To account for this effect, we associate different probabilities with different cluster types, namely $f_{\mathrm{GC}}$ for globular, $f_{\mathrm{YC}}$ for young and open clusters, and $f_{\mathrm{NC}}$ for nuclear clusters. These quantities are defined in such a way that $f_{\mathrm{GC}}+f_{\mathrm{YC}}+f_{\mathrm{NC}}=1$. This choice implies, for example, that a given sample of dynamical mergers will contain a fraction $f_{\mathrm{GC}}$ of mergers originated in globular clusters.

To initialize $f_{\mathrm{GC}}, f_{\mathrm{YC}}$, and $f_{\mathrm{NC}}$, we take advantage of the most recent results connected with dynamical BBH mergers.

As discussed above, the most recent models suggest that mergers developing in globular clusters can outnumber those forming in nuclear cluster by a factor of up to 3-5. Our knowledge of the merger rate from young star clusters is more uncertain, because massive stars form preferentially in young star clusters (Portegies Zwart et al. 2010). Hence, young star clusters can provide a large fraction of the $\mathrm{BBH}$ mergers that occur in the field (see, e.g., Bouffanais et al. 2019; di Carlo et al. 2019a).

Based on these speculations, we assume $\left(f_{\mathrm{GC}}, f_{\mathrm{YC}}, f_{\mathrm{NC}}\right)=$ $(0.6,0.2,0.2)$ as a fiducial value. Nevertheless, it must be noted that these numbers rely upon a number of unknown parameters, like the number of young and globular clusters in a given cluster, the merger efficiency (i.e., the number of mergers per unit of cluster mass), and the cluster metallicity distribution. We investigate how these quantities affect the results in Section 4.

Another intriguing feature of dynamical mergers is the mass ratio. The most massive BHs quickly segregate to the host cluster center and tend to pair together. This can lead to the preferential formation of BBHs with high mass ratios, regardless of the cluster type (Downing et al. 2010; Amaro-Seoane \& Chen 2016; Rodriguez et al. 2016; Arca Sedda \& Benacquista 2019; di Carlo et al. 2019a). In order to account for this effect, we assume that dynamical mergers have mass ratios above a minimum value $q_{\min }$.

\subsection{Single and BBH Natal Mass and Spin}

In order to calculate the mass of $\mathrm{BBH}$ components (for isolated binaries) and of single $\mathrm{BHs}$ (for dynamical binaries), we take advantage of the MOBSE (Giacobbo et al. 2018) population synthesis code. The code is an upgraded version of the BSE (Hurley et al. 2002) stellar evolution package, which allows the user to follow the evolution of binary and single stars from their birth down to the final stages of evolution. The main distinctive feature of MOBSE with respect to other population-synthesis codes descending from BSE is that mass loss by stellar winds in MOBSE depends on both the metallicity and the stellar luminosity of a massive star: the closer the stellar luminosity gets to the Eddington ratio, the higher the mass loss, regardless of its metallicity. In addition, MOBSE includes a treatment of pair instability and pulsational pair instability supernovae (Spera \& Mapelli 2017; Woosley 2017). In the following, we make use of model CC15 $\alpha 1$, presented in Giacobbo et al. (2018), which assumes low natal kicks for both core-collapse and electron-capture supernovae.

In order to cover the span of metallicity typical of metal-rich and metal-poor systems, we create 12 different single and BBH populations, characterized by $Z$ values between $Z=0.0002$ and $Z=0.02 \equiv Z_{\odot}$.

To create the sample of isolated BBHs, we first generate the binary stars following Giacobbo et al. (2018). The primary star mass is selected according to a Kroupa (2001) mass function truncated between 5 and $150 M_{\odot}$; the mass ratio is thus extracted according to $P(q) \propto q^{-0.1}$ to obtain the secondary star mass. The binary period is assigned according to $P(\tau) \propto \tau^{-0.5}$, assuming limiting values of $\tau \equiv \log (P /$ day $)=0.15-5.5$, whereas the eccentricity is drawn between 0 and 1 according to $P(e) \propto e^{-0.42}$. Note that the assumptions above are motivated by observations of Galactic O-type stars (Sana et al. 2012). From the whole sample of binaries modeled with MOBSE, we retain only those whose product is a BBH for which the sum of the time needed for the two stars to become a BBH and the merger time is smaller than 14 Gyr. For a description of the resulting BBH mass spectrum, see Giacobbo et al. (2018).

For dynamical BBHs, we draw the mass of each progenitor star according to a Kroupa (2001) mass function within the same range of values assumed for isolated binaries. The natal mass of $\mathrm{BHs}$ is calculated via MOBSE, the two BHs in dynamical BBHs are randomly paired following a uniform distribution between the minimum mass ratio $q_{\min }$ (which depends on the considered model, as described in the previous section) and the maximum mass ratio $q=1$. The probability to randomly draw a $\mathrm{BBH}$ from the isolated or the dynamical samples is then weighted with the assumed observational bias (see Section 2.2).

Determining BH natal spins represents an issue that is still largely debated in the stellar evolution community. Some recent work has proposed a relation between the spin amplitude and the mass of the stellar carbon-oxygen core (e.g., Belczynski et al. 2020). According to this prescription, BHs with natal masses $\leqslant 40 M_{\odot}$ have natal spins above 0.8 , with a slight dependence on the progenitor metallicity, while the spin decreases at increasing the BH mass. In opposition to this, other studies propose that massive stellar progenitors undergo 
an efficient angular momentum loss that leads the $\mathrm{BH}$ to have a spin $\sim 0.075-0.04$ times the inverse of the mass, at least for BHs heavier than $30 M_{\odot}$ (Amaro-Seoane \& Chen 2016). The situation is even more complex if BHs form in a binary. In order to cope with our ignorance about the processes that regulate BHs natal spin amplitude, we assume a uniform distribution of spins between 0 and 1 in both isolated and dynamical binaries.

Another crucial point is related to spin orientation. Spin alignment directly affects the remnant $\mathrm{BH}$ final spin amplitude. In the case of dynamical BBHs, the spin orientation is expected to be isotropically distributed, $P(\theta)=$ const. On the other hand, predicting the alignment of isolated BBHs is more complex. In principle, one can expect that the mutual tidal field exerted from one component to the other would somehow maintain the spins aligned. However, during the stages that lead a star to turn into a $\mathrm{BH}$, there are several processes (such as supernova explosion) that can drive the spin reorientation. Following Arca Sedda \& Benacquista (2019), we control the level of spin misalignment assuming that the angle between the two spins, $\theta$, is characterized by a distribution $P(\cos \theta)=k\left(n_{\theta}\right)(\cos \theta+1)^{n_{\theta}}$. This choice implies that increasing $n_{\theta}$ values correspond to more aligned distribution, with $n_{\theta}=0(\infty)$ corresponding to an isotropic (fully aligned) distribution.

In our fiducial model, we assume $n_{\theta}=0$, which corresponds to isotropically oriented spins, i.e., the same as for dynamical binaries.

\subsection{Black Hole Remnant Final Mass and Spin}

During the merger, part of the binary mass is radiated away in the form of GWs, ergo the final $\mathrm{BH}$ mass $M_{\text {rem }}$ will be a fraction of the progenitor $\mathrm{BBH}$ mass $M_{\mathrm{BBH}}$. Using the LVC data catalog (GWTC-1, Abbott et al. 2019a), we find that the $M_{\mathrm{rem}}-M_{\mathrm{BBH}}$ is excellently described by a linear relation of the form

$$
M_{\mathrm{rem}}=A M_{\mathrm{BBH}},
$$

where $A=0.954 \pm 0.002$. Although tantalizing, calculating the remnant $\mathrm{BH}$ mass via this relation can neglect some important dependencies. A rigorous approach would require numerical relativity, which allows us to follow the last stages of $\mathrm{BBH}$ evolution and infer crucial information on the GWs produced during the $\mathrm{BBH}$ inspiral, merger, and ringdown (Pretorius 2005; Baker et al. 2006; Campanelli et al. 2006; Sperhake 2015). Therefore, we calculate $M_{\text {rem }}$ by taking advantage of the fitting procedure described in Jiménez-Forteza et al. (2017), although this is tailored to aligned-spin binaries based on numerical relativity simulations. We use the same approach to calculate the remnant spin $a_{\text {rem }}$, making use of the so-called "augmentation" technique (Hughes \& Blandford 2003; Rezzolla et al. 2008), which allows us to include the in-plane spin components in $a_{\text {rem }}$ calculation. As a cross-check, we also calculate $a_{\text {rem }}$ using the fitting formulae provided by Hofmann et al. (2016), following our previous paper (Arca Sedda \& Benacquista 2019). We note that the $M_{\mathrm{BBH}}-M_{\text {rem }}$ relation calculated for a sample of over $10^{5}$ models is exquisitely described by a linear relation with slope $A=0.934 \pm 0.001$, regardless of the spin orientation, $\mathrm{BBH}$ mass ratio, or total mass.

\subsection{GW Recoil}

Promptly after the merger, the remnant receives a kick due to anisotropic GW emission, the amplitude of which can exceed $10^{3} \mathrm{~km} \mathrm{~s}^{-1}$. Nonetheless, the potential well of the heaviest clusters (globular and nuclear clusters) might be sufficiently deep to retain some of the post-merger BHs. This allows the remnant BHs to possibly undergo multiple mergers (Miller \& Hamilton 2002; Fishbach \& Holz 2017; Gerosa \& Berti 2017; Antonini et al. 2019; Rodriguez et al. 2018; Arca Sedda \& Benacquista 2019; Kimball et al. 2020; Doctor et al. 2020; Rodriguez et al. 2019), leading to higher and higher $\mathrm{BH}$ masses. The probability for BHs to undergo at least two mergers can rise up to $\sim 40 \%$ for both globular (Rodriguez et al. 2018) and nuclear clusters (Antonini et al. 2016). To account for multiple mergers, in our model we calculate the GW recoil kick as (Campanelli et al. 2007; Lousto \& Zlochower 2008; Lousto et al. 2012)

$$
\begin{gathered}
\boldsymbol{v}_{k}=v_{m} \hat{e}_{\perp, 1}+v_{\perp}\left(\cos \xi \hat{\boldsymbol{e}}_{\perp, 1}+\sin \xi \hat{\boldsymbol{e}}_{\perp, 2}\right)+v_{\|} \hat{\boldsymbol{e}}_{\|}, \\
v_{m}=A \eta^{2} \sqrt{1-4 \eta}(1+B \eta), \\
v_{\perp}=\frac{H \eta^{2}}{1+q_{\mathrm{BBH}}}\left(a_{2, \|}-q_{\mathrm{BBH}} a_{1, \|}\right), \\
v_{\|}=\frac{16 \eta^{2}}{1+q_{\mathrm{BBH}}}\left[V_{11}+V_{A} \Xi_{\|}+V_{B} \Xi_{\|}^{2}+V_{C} \Xi_{\|}^{3}\right] \\
\times\left|\boldsymbol{a}_{2, \perp}-q_{\mathrm{BBH}} \boldsymbol{a}_{1, \perp}\right| \cos \left(\phi_{\Delta}-\phi_{1}\right) .
\end{gathered}
$$

Here, $\eta \equiv q_{\mathrm{BBH}} /\left(1+q_{\mathrm{BBH}}\right)^{2}$ is the symmetric mass ratio, while $\boldsymbol{\Xi} \equiv 2\left(\boldsymbol{a}_{2}+q_{\mathrm{BBH}}^{2} \boldsymbol{a}_{1}\right) /\left(1+q_{\mathrm{BBH}}\right)^{2}$. The subscripts $\perp$ and $\|$ mark the perpendicular and parallel direction of the $\mathrm{BH}$ spin vector with respect to the direction of the $\mathrm{BBH}$ angular momentum. The unit vectors $\left(\hat{e}_{\|}, \hat{e}_{\perp, 1}, \hat{e}_{\perp, 2}\right)$ constitute an orthonormal basis with one component directed perpendicular to $\left(\hat{e}_{\|}\right)$and two components lying in the $\mathrm{BBH}$ orbital plane. We set $A=1.2 \times 10^{4} \mathrm{~km} \mathrm{~s}^{-1}, \quad B=-0.93, \quad H=6.9 \times 10^{3} \mathrm{~km} \mathrm{~s}^{-1}$, and $\xi=145^{\circ}$ (see González et al. 2007; Lousto \& Zlochower 2008), and $\quad V_{A, B, C}=(2.481,1.793,1.507) \times 10^{3} \mathrm{~km} \mathrm{~s}^{-1}$ (Lousto et al. 2012). Here, $\phi_{\Delta}$ represents the angle between the direction of the infall at merger (which we randomly draw in the BBH orbital plane) and the in-plane component of $\boldsymbol{\Delta} \equiv$ $\left(m_{1}+m_{2}\right)^{2}\left(\boldsymbol{a}_{2}-q_{\mathrm{BBH}} \boldsymbol{a}_{1}\right) /\left(1+q_{\mathrm{BBH}}\right)$, while $\phi_{1}=0-2 \pi$ is the phase of the $\mathrm{BBH}$, extracted randomly between the two limiting values.

According to the above equations, the GW recoil kick imparted to the merger remnant can vary between $\sim 10$ and $3000 \mathrm{~km} \mathrm{~s}^{-1}$, higher than the typical velocity dispersion of both open $(\sigma \simeq$ $\left.1-5 \mathrm{~km} \mathrm{~s}^{-1}\right)$ and globular clusters $\left(\sigma \simeq 10-15 \mathrm{~km} \mathrm{~s}^{-1}\right)$. Note that we refer to the value of $\sigma$ calculated at the cluster half-mass radius, though it can be much higher in the inner core, especially if the cluster hosts a central massive black hole or a stellar black hole cusp. In the case of nuclear clusters, the velocity dispersions of which can have escape velocities $\sigma \geqslant(1-3) \times 10^{2} \mathrm{~km} \mathrm{~s}^{-1}$ (see, for instance, Georgiev et al. 2009), the chance for a post-merger $\mathrm{BH}$ to be retained in the host cluster and undergo a further merger is not negligible. For each $\mathrm{BBH}$, we calculate the GW recoil $\left|v_{k}\right|$ via Equations (2)-(5), and we allow the 


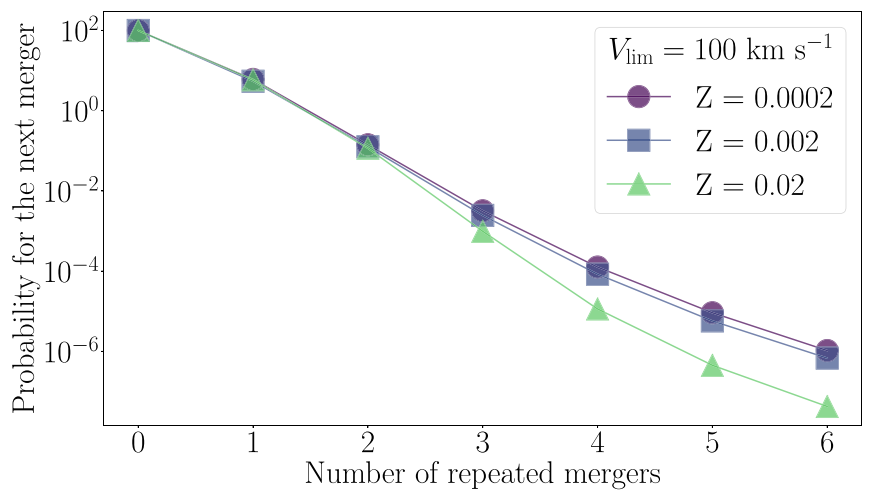

Figure 3. Probability for a $\mathrm{BH}$ merger to undergo another merger as a function of the number of mergers that the $\mathrm{BH}$ has already experienced. We assume that the remnant is ejected from the parent cluster if the GW velocity exceeds $V_{\text {lim }}=100 \mathrm{~km} \mathrm{~s}^{-1}$. We consider metallicity $Z=0.0002$ (purple circles), 0.002 (blue squares), and 0.02 (green triangles).

remnant to undergo another merger if $\left|\boldsymbol{v}_{k}\right|<v_{\max }$, where $v_{\max }=$ $(3,15,100) \mathrm{km} \mathrm{s}^{-1}$ for young, globular, and nuclear clusters, respectively.

Figure 3 shows the combined probability, for a total of $10,000 \mathrm{BBHs}$ harbored in a nuclear cluster, to receive a $\mathrm{GW}$ kick below $100 \mathrm{~km} \mathrm{~s}^{-1}$ and undergo a further merger, assuming a Kroupa initial mass function (Kroupa 2001) for the progenitor stars and calculating the $\mathrm{BH}$ natal mass according to the single $\mathrm{BH}$ mass spectrum described in the previous section (Giacobbo et al. 2018). After the first merger, the remnant $\mathrm{BH}$ has a chance of $\sim 5 \%-10 \%$ to be retained. At each stage, the probability is calculated as the product of all the previous ones, and decreases by roughly one order of magnitude at any successive stage.

\section{Results}

In this section, we use the fiducial model to infer a population of merging BBHs. We also discuss how different assumptions impact the remnant mass and spin distributions.

\subsection{Fiducial Model}

The top panel of Figure 4 shows the final mass and spin distribution of $10^{5}$ mergers calculated upon the assumptions listed above. For comparison's sake, we overlap the ten BBH mergers detected by the LVC in the first and second observing runs (see Table 3 in Abbott et al. 2019a).

The remnant mass is characterized by a complex distribution that shows two peaks at roughly $\sim 18 M_{\odot}$ and $\sim 25 M_{\odot}$, along with a broader component peaking at $M_{\text {rem }} \sim 55 M_{\odot}$. The spin distribution peaks at $a_{\text {rem }} \sim 0.7$, with a FWHM $\simeq 0.1$. Comparing our $M_{\text {rem }}-a_{\text {rem }}$ plane with actual detections, it is apparent how BHs with both low mass (i.e., $\lesssim 20 M_{\odot}$ ) and high mass fall in the maximum likelihood of our distribution. Only the most massive BH detected so far, GW170729, seems to lie out of the main distribution, showing both larger mass and spin compared to the overall distribution. This might suggest a peculiar formation history. In Section 3.4, we discuss one possible route to the formation of GW170729-like sources as the result of multiple mergers in dense star clusters. The central panel in Figure 4 compares the total mass $\left(M_{\mathrm{BBH}}\right)$ and mass ratio $\left(q_{\mathrm{BBH}}\right)$ of our BBHs with $\mathrm{O} 1$ and $\mathrm{O} 2 \mathrm{LVC}$ detections. We also compare our model with observed chirp mass $\left(\mathcal{M}_{\mathrm{BBH}}\right)$ and effective spin parameter $\left(\chi_{\text {eff }}\right)$, defined as

$$
\begin{gathered}
\mathcal{M}_{\mathrm{BBH}}=\left(m_{1} m_{2}\right)^{3 / 5} /\left(m_{1}+m_{2}\right)^{1 / 5}, \\
\chi_{\mathrm{eff}}=\left(a_{1} \cos \theta_{1}+q_{\mathrm{BBH}} a_{2} \cos \theta_{2}\right) /\left(1+q_{\mathrm{BBH}}\right),
\end{gathered}
$$

where $\theta_{i}$ is the angle between the $\mathrm{BBH}$ angular momentum vector and the direction of the spin of the $i$ th $\mathrm{BH}$. As shown in the bottom panel of Figure 4, all the LVC sources fall inside the region enclosing $70 \%$ of our models, with the only exception being GW170729.

We note that single BHs can have mass up to $\sim 65 M_{\odot}$ in the simulations of Giacobbo et al. (2018), while BHs in isolated binaries that reach coalescence within a Hubble time have a maximum mass of $\sim 45 M_{\odot}$. Hence, the seeming dearth of remnants with a final mass above $80 M_{\odot}$ is due to a combination of factors: i) the population is dominated by isolated binaries, which constitute the $67 \%$ of mergers in our fiducial model, and only $\mathrm{BHs}$ with mass $<45 M_{\odot}$ coalesce in the isolated binary sample; ii) as shown in Figure 2, the assumption of a cluster metallicity distribution flat in the logarithm implies that only roughly half of dynamical mergers have a metallicity below $\sim 0.1 Z_{\odot}$, i.e., smaller enough to trigger the formation of $\mathrm{BHs}$ heavier than $40-50 M_{\odot}$.

Metallicity is one of the most important ingredients in determining the remnant $\mathrm{BH}$ mass. Figure 5 shows the $M_{\text {rem }}$ distribution for different metallicity bins. The mass distribution at the lowest metallicities shows an evident peak toward values $M_{\text {rem }} \sim 50-80 M_{\odot}$, and an interesting tail to values exceeding $100 M_{\odot}$. The high end of this distribution becomes fainter and fainter at increasing $Z$ values, while at the same time the overall distribution becomes bimodal, acquiring a sizeable population of BHs with masses in the $10-30 M_{\odot}$ mass range. At metallicities above $Z \geqslant 0.008$, the whole distribution shifts toward lower $M_{\text {rem }}$ values and the high-end tail truncation value of the distribution progressively decreases, reaching $M_{\text {rem,max }} \sim 80 M_{\odot}$ at solar metallicity.

Unlike the remnant mass, the remnant spin distribution does not show any appreciable dependence on the metallicity, due to the assumption that $\mathrm{BH}$ natal spin amplitude is independent of the metallicity or the $\mathrm{BBH}$ formation channel.

\subsection{Matching $\mathrm{O} 1+O 2$}

Using our fiducial model, we now quantify the probability to obtain the currently known population of GW sources with our method. We create a sample of 100,000 mergers for which we store total mass and mass ratio. To quantify the matching between observations and modeled binaries, we define two different comparison strategies. In the first, for each LVC source, ${ }^{11}$ we calculate the fraction of modeled BBHs having a total mass within $30 \%$ of the observed value. For instance, in the case of GW170104, we find that nearly $57.1 \%$ of the modeled mergers have a total mass within $30 \%$ of the observed value, i.e., $\sim 50 M_{\odot}$. In the second, we calculate the fraction of modeled BBHs that have both a total mass and a mass ratio within $30 \%$ of the observed value, ${ }^{12}$ i.e., $35<M_{\mathrm{BBH}} / M_{\odot}<65$ and $0.45<q_{\mathrm{BBH}}<0.85$ in the case of GW170104.

Figure 6 shows these probabilities for the current population of 10 LVC sources. We find that our fiducial model can match the mass of all $\mathrm{O} 1+\mathrm{O} 2$ BBHs. For instance, mergers with mass

\footnotetext{
${ }^{11}$ Data are taken from https://www.gw-openscience.org/; see also Abbott et al. (2019a).

12 Note that the error associated to the observed quantities in some cases exceeds $30 \%$, especially for the observed mass ratio.
} 

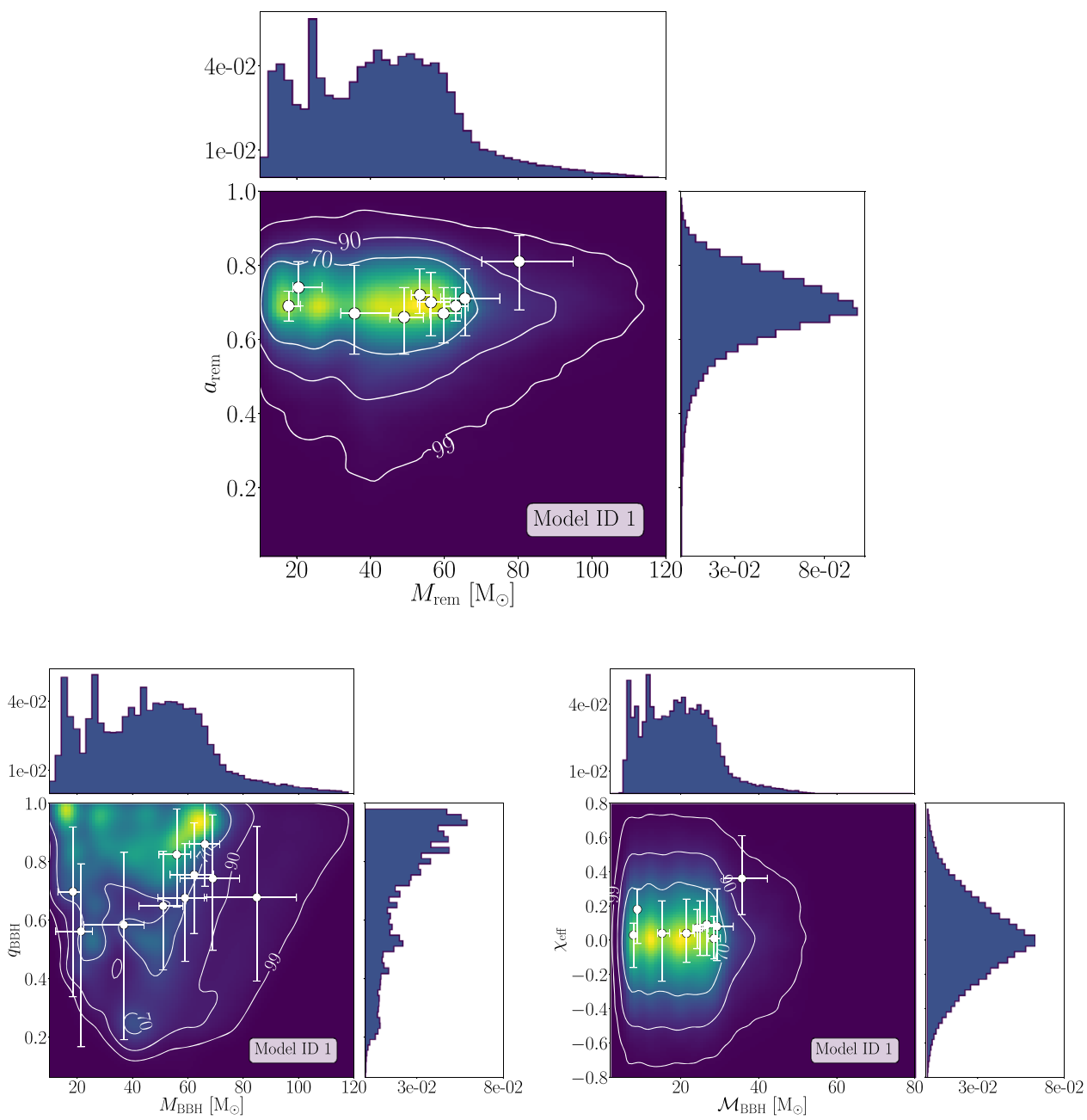

Figure 4. Top panel: remnant mass and spin. Bottom panels: BBH total mass $M_{\mathrm{BBH}}$ and mass ratio $q_{\mathrm{BBH}}$ (left panel), and BBH chirp mass $\mathcal{M}_{\mathrm{BBH}}$ and effective spin parameter $\chi_{\text {eff }}$ (right panel). All panels refer to the fiducial model (SET ID 1). Colored map represents the normalized density in each bin, smoothed with a Gaussian kernel density estimation. Contour lines encompass $70 \%, 90 \%$, and $99 \%$ of the total sample, respectively.

and mass ratio similar to the first observed source, GW150914, have a $\sim 20 \%$ probability to be selected in our fiducial model. The probability raises up to $\sim 40 \%$ if we limit the comparison to the BBH mass only. The matching probability is even larger for sources with masses in the range $50-65 M_{\odot}(\mathrm{GW} 170104$, GW170809, GW170814), while it drops to $\sim 5 \%-10 \%$ when applied to the lightest mergers, $M_{\mathrm{BBH}} \lesssim 20 M_{\odot}$.

\subsection{Massive BH Remnants}

One of the most interesting features of our BBH merger products is the possible formation of BHs with masses in the IMBH mass range, i.e., $\sim 10^{2} M_{\odot}$. In order to understand the frequency of the formation of such massive merger products in our fiducial model, in Figure 7 we show the distribution of remnant masses for $10^{5} \mathrm{BBHs}$ created according to models ID 1 (fiducial), 3a (isolated with $P(Z)=$ SDSS), 3c (isolated with $P(\log Z)=$ const), and $4 \mathrm{a}$ (dynamical).

Our fiducial model predicts up to five merger products heavier than $\sim 93 M_{\odot}$ and at least one with mass above $\sim 114 M_{\odot}$ out of $100 \mathrm{BBH}$ mergers in the local universe. Observing such a massive $\mathrm{BH}$ would provide us with a window into the lowest metallicities, and could represent an exquisite signature of dynamical formation. Indeed, according to our model, remnant BHs with masses above $80-90 M_{\odot}$ come from BBHs formed in metal-poor environments, ${ }^{13}$ where the contribution of isolated binaries is relatively small, due to the metallicity distribution in the local universe (see also Figure 5).

Compared to the fiducial model, the $M_{\text {rem }}$ distribution for dynamical models (4a) shows a tail that extends to larger values of the remnant mass. If dynamical mergers dominate the global population of $\mathrm{BBH}$ mergers, this model predicts at least $1 \mathrm{BH}$ remnant with mass beyond $140 M_{\odot}$ out of 100 mergers. In contrast, isolated models (ID $3 \mathrm{a}$ and $3 \mathrm{c}$ ) produce a narrower distribution of $M_{\mathrm{rem}}$, limited to roughly $75-80 M_{\odot}$, a limit set by the choice of the binary stellar evolution recipes implemented in MOBSE. Given the evident differences in the merger mass distribution among dynamical and isolated binaries, we calculate the percentage of BBHs with remnant mass in the range $\leqslant 35 M_{\odot}, 35-50 M_{\odot}, 50-75 M_{\odot}$, and $\geqslant 75 M_{\odot}$ for models ID $1,3 \mathrm{a}, 3 \mathrm{c}$, and $4 \mathrm{a}$.

Table 2 summarizes the percentage of BBHs with a remnant mass in different mass ranges and for different models. Comparing the fiducial (ID 1), isolated only (ID 3a and 3c), and dynamical

\footnotetext{
$\overline{13}$ It must be noted that such massive $\mathrm{BHs}$ can also form as the product of massive main-sequence stars' collisions (Spera et al. 2019). In dense clusters, BHs forming via this channel can capture a companion and lead to the formation of even heavier BHs through coalescence, as shown by di Carlo et al. (2019a).
} 


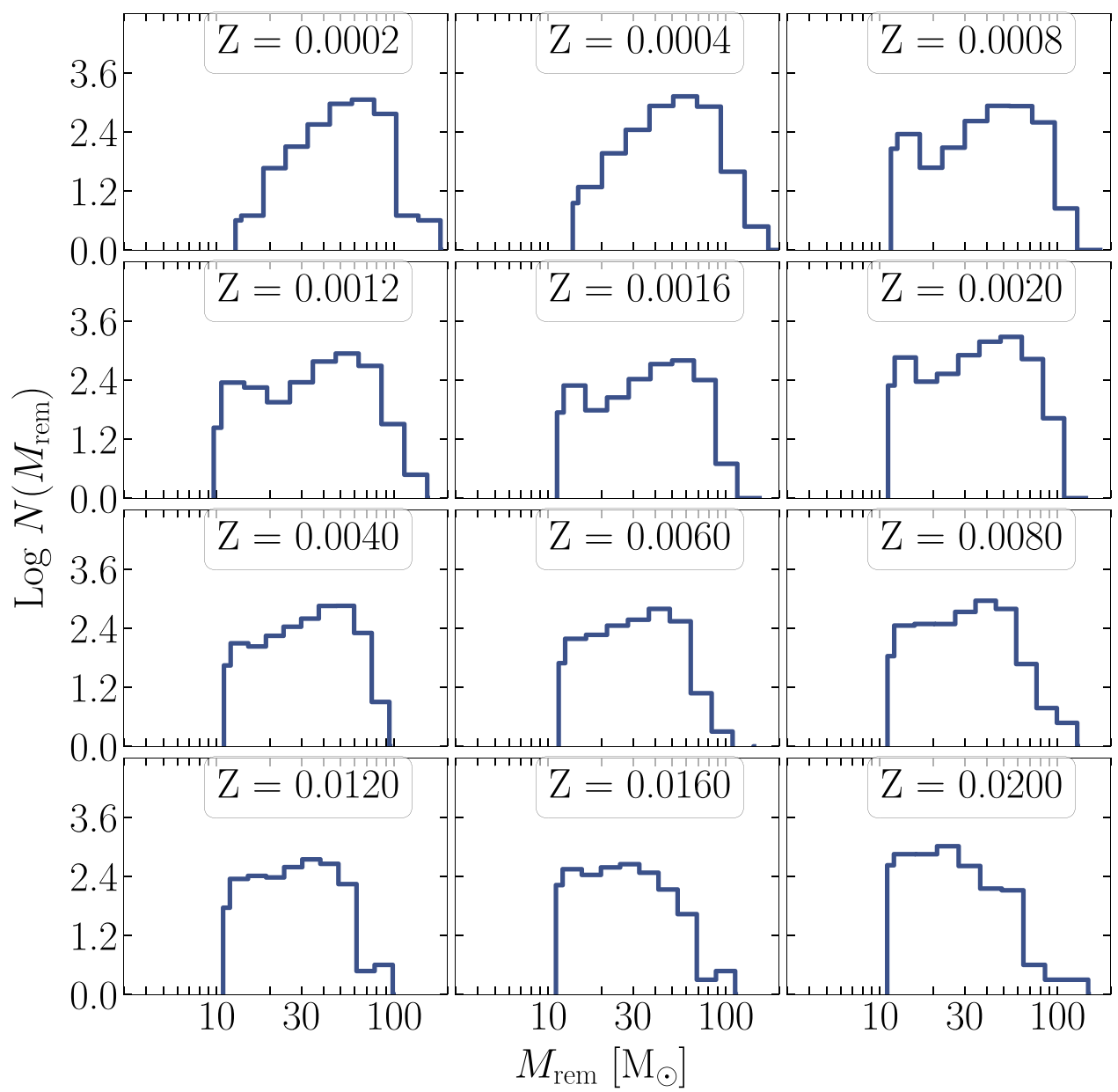

Figure 5. Remnant final mass for different metallicity bins and for the fiducial model. Metallicity increases from top to bottom and from left to right.

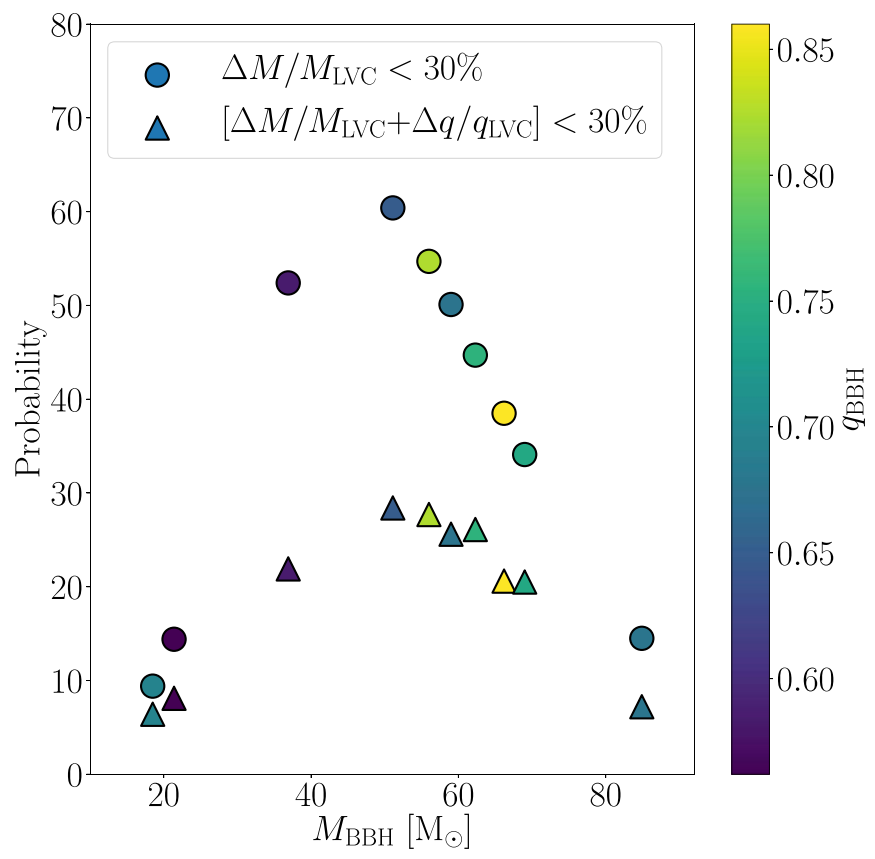

Figure 6. Probability to select a BBH compatible with one of the 10 confirmed LVC BBHs from O1 and O2 (Abbott et al. 2019b). Each circle or triangle corresponds to one LVC BBH. The color coding marks LVC BBH mass ratios. Triangles (circles) represent the probability to draw-from the fiducial model sample - a merger with mass ratio and total mass (total mass only) within $30 \%$ of the corresponding observed value. only (ID 4a) models makes apparent a striking difference between the predicted percentage of binaries with either low $\left(<30 M_{\odot}\right)$ or high mass $\left(\geqslant 70 M_{\odot}\right)$. The models in which we assume that the merger population is mostly composed of isolated binaries (ID 3a and 3c) predict $\sim 65 \%-75 \%$ of mergers with $M_{\text {rem }}<50 M_{\odot}$, and almost no binaries with $M_{\mathrm{rem}} \geqslant 75 M_{\odot}$. The percentage of mergers with masses falling in the low-end and high-end tails of the mass distribution can be extremely useful to place constraints on the contribution of dynamical mergers to the overall population. In the fiducial model, for instance, we find that the percentage of mergers lying in the high-end tail of the mass distribution $\left(P_{\geqslant 75}=5.8 \%\right)$ is $\sim 1 / 6$ of the percentage of mergers lying in the low-end tail of the distribution $\left(P_{\geqslant 35}=36.1 \%\right)$.

In model 4a, where dynamical mergers dominate the overall population, the percentage of mergers with masses $>50 M_{\odot}$ $(52 \%)$ and $<50 M_{\odot}(48 \%)$ is very similar, and the heaviest mergers $\left(P_{\geqslant 75}=16.8 \%\right)$ are $64 \%$ of the lowest mass BBHs $\left(P_{\leqslant 35}=26.1 \%\right)$. Therefore, we expect that:

1. if more than $60 \%$ of BBH mergers have mass $<50 M_{\odot}$, the isolated channel outweighs the dynamical one;

2. the absence of remnants with masses above $75 M_{\odot}$ would imply a negligible contribution of dynamical mergers to the overall population;

3. a comparable number of mergers with $M_{\mathrm{rem}}<35 M_{\odot}$ and $M_{\text {rem }} \geqslant 75 M_{\odot}$ suggests that dynamical binaries are the majority of the overall population. 

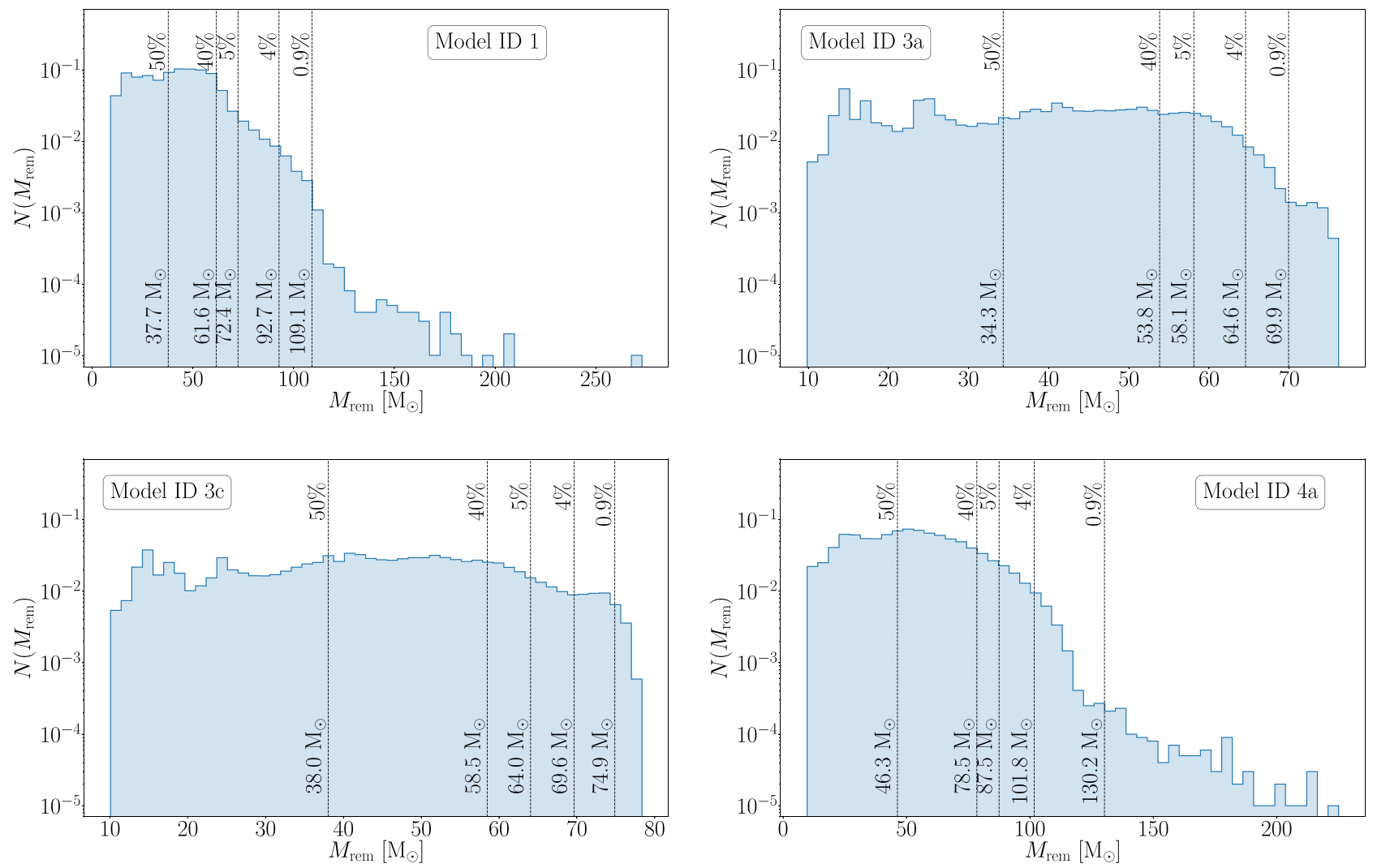

Figure 7. Remnant mass distribution for $10^{5} \mathrm{BBH}$ mergers in the fiducial model (ID 1, top left panel), model 3a (top right panel), model 3c (bottom left panel), and model 4a (bottom right panel). Labels indicate the percentage of binaries with remnant mass in a given mass range. Out of $100 \mathrm{BBHs}$, the fiducial model predicts five mergers with remnant mass $M_{\text {rem }}>72 M_{\odot}$ and $\sim 1$ with mass above $\sim 93 M_{\odot}$.

Table 2

Percentage of Mergers with a Remnant Mass in Different Mass Ranges

\begin{tabular}{lrrrrr}
\hline \hline ID & $N_{\text {obs }}$ & $\begin{array}{c}P_{<35} \\
(\%)\end{array}$ & $\begin{array}{c}P_{35-50} \\
(\%)\end{array}$ & $\begin{array}{c}P_{50-75} \\
(\%)\end{array}$ & $\begin{array}{r}P_{>75} \\
(\%)\end{array}$ \\
\hline 1 & 100 & 36.1 & 28.0 & 30.1 & 5.8 \\
$3 \mathrm{a}$ & 100 & 41.9 & 30.8 & 27.2 & 0.0 \\
$3 \mathrm{c}$ & 100 & 33.2 & 31.5 & 34.7 & 0.6 \\
4a & 100 & 26.1 & 21.9 & 35.2 & 16.8 \\
LVC & 10 & 20.0 & 20.0 & 50.0 & 10.0 \\
\hline
\end{tabular}

As shown in Table 2, 60\% of the 10 confirmed BBHs in O1 and $\mathrm{O} 2$ have merger remnants heavier than $50 M_{\odot}$ (Abbott et al. $2019 \mathrm{~b})$, showing an interesting similarity with our model $4 \mathrm{a}$ (dynamical mergers only).

\subsection{A Multimerger Route to the Formation of GW170729-like Sources}

One of the most interesting sources detected by the LVC is GW170729, a BBH merger that left behind a highly spinning $\left(a_{\text {rem }}=0.81_{-0.13}^{+0.07}\right)$ and massive $\left(M_{\text {rem }}=80.3_{-10.2}^{+14.6} M_{\odot}\right)$ BH. Compared to the global distribution of mergers shown in Figure 4, sources of this kind have a relatively low probability to form. Indeed, less than $10 \%$ of the simulated sources in the fiducial model have $M_{\text {rem }}>80 M_{\odot}$.

One possibility is that GW170729 formed dynamically, possibly from a progenitor that underwent multiple mergers. In the following, we use our machinery to test this hypothesis.
We use the BH mass spectrum from MOBSE to select the initial mass of two BHs, assuming that they merge inside a star cluster. We select the primary $\mathrm{BH}$ mass in the range $10-65 M_{\odot}$, while the companion mass is extracted assuming a flat mass ratio distribution. Spins for both BHs are drawn with uniform amplitude between 0 and 1 and with isotropically distributed orientations. We calculate the remnant mass and spin, and associate a new companion from the same mass spectrum to mimic a second merger. To mimic a sequence of mergers, we repeat this procedure $n_{\text {mer }}$ times, assuming three values for the metallicity $(Z=0.0002,0.002,0.02)$. For each $Z$ value, we create 10,000 merger trees, and for each $n_{\text {mer }}$ value, we calculate the mean mass $\left(\left\langle M_{\mathrm{rem}}\right\rangle\right)$ and spin $\left(\left\langle a_{\mathrm{rem}}\right\rangle\right)$ of the remnant $\mathrm{BH}$. Moreover, we calculate the probability to form a remnant $\mathrm{BH}$ with a mass, or spin, within $30 \%$ of the observed value for GW170729. These quantities are shown in Figure 8. The upper box in the top panel highlights that $\left\langle M_{\mathrm{rem}}\right\rangle$ values compatible with GW170729 can be achieved with 1-2 mergers in the metallicity range $0.0002-0.002$, while at least 5-6 repeated mergers are needed to explain such large mass in an environment characterized by a solar metallicity. Indeed, as shown in the lower box of the top panel, the probability to form a BH with remnant mass close to GW170729 is $~ 40 \%-60 \%$ if the number of mergers is $n_{\text {mer }}=1-2$ and the metallicity is low $(Z<0.002)$, while for solar metallicities, the probability ranges between $20 \%$ and $60 \%$ assuming $n_{\text {mer }}=5$ or 6 , respectively. The average remnant spin, as shown in the lower panel of the figure, exhibits a peak at $n_{\text {mer }}=1-2$, regardless of the metallicity, where $\left\langle a_{\text {rem }}\right\rangle \sim 0.65$, i.e., slightly off the observational error. This quantity reduces as we increase the 

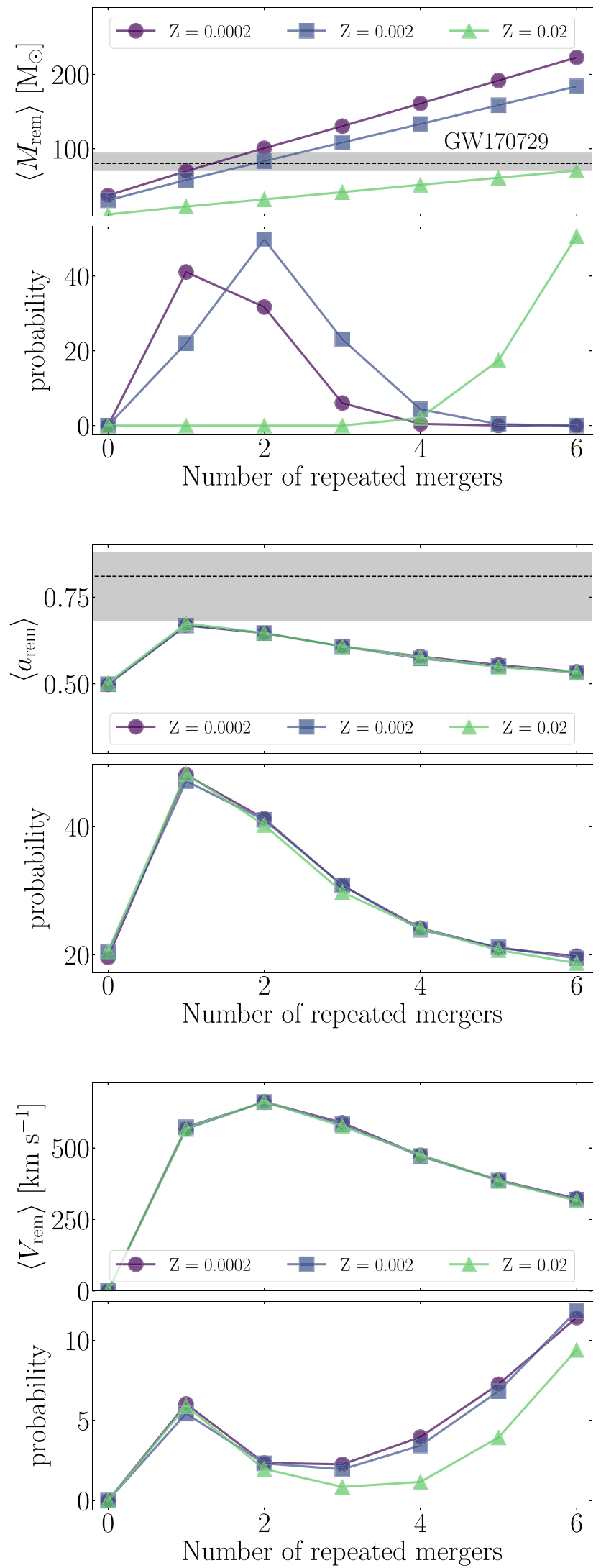

Figure 8. Top panel: remnant BH mass (upper box) and matching probability for GW170729 (lower box) as a function of the number of mergers. Shaded area encompasses the $90 \%$ credible interval of the GW170729 mass. Central panel: same as above, but for the remnant BH spin. Bottom panel: average GW recoil velocity for all modeled mergers (upper box) and probability that the kick remains below $100 \mathrm{~km} \mathrm{~s}^{-1}$ (lower box), thus allowing a next-generation merger. number of mergers, thus limiting the possibility for GW170729 to have originated through more than two subsequent mergers.

Furthermore, the post-merger recoil kick can eject the remnant $\mathrm{BH}$ outside the star cluster. Assuming that the progenitors of GW170729 formed in a nuclear cluster, we have calculated the recoil velocity $v_{k}$ and the probability that $v_{k}<v_{\max } \equiv 100 \mathrm{~km} \mathrm{~s}^{-1}$ for each $\mathrm{BBH}$ and for each merger. The upper box of the bottom panel in Figure 8 shows the average recoil kick received at the last merger, while the lower box indicates the probability that such velocity falls below $100 \mathrm{~km} \mathrm{~s}^{-1}$. Looking at the bottom box, we see that there is a $5 \%$ probability for a $\mathrm{BH}$ formed via a "first generation" merger to get a kick below $100 \mathrm{~km} \mathrm{~s}^{-1}$, whereas this probability falls to $\sim 2 \%$ if the $\mathrm{BH}$ is originated via two subsequent mergers. To determine the combined probability for a $\mathrm{BH}$ to undergo a series of mergers, we need to multiply the probability to be retained at every step. This implies that, in a typical NC, the probability for a $\mathrm{BH}$ to be retained after one merger event is around $\sim 5 \%$, whereas the probability to be retained after two successive mergers is around $5 \% \times 2 \%=0.1 \%$.

Figure 8 suggests that GW170729 likely formed in a metalpoor environment $(Z<0.002)$, such as a dense globular cluster, via either a single merger or two subsequent mergers.

\section{Discussion: Quantifying the Uncertainties}

In this section, we discuss how BBH formation channels, distribution of galaxy metallicity, and merger probabilitymetallicity correlation affect our results.

\subsection{Impact of Metallicity}

Metallicity is one of the parameters that most influences the merger remnant mass and spin distribution. As discussed in previous sections, the ansatz behind our fiducial model is that the metallicity distribution of merging BBHs depends on the formation channel, and that a larger merger probability corresponds to a lower metallicity.

In order to quantify the role of metallicity in shaping the $M_{\text {rem }}-a_{\text {rem }}$ plane, let us assume that the merger probability does not depend on the metallicity, namely that the average number of mergers in different metallicity bins is nearly constant, $f(Z)=$ const. This implies that the metallicity distribution of galaxies in the local universe provides a one-to-one match to the metallicity of merging BBHs. ${ }^{14}$

Under this assumption, we explore three different cases. In the first case, we assume that star clusters and host galaxies are characterized by the same metallicity distribution, regardless of the clusters' type (set ID 2a). In the second case, we assume that metallicity of globular and nuclear clusters is equally distributed in logarithmic bins, while the open clusters and the Galaxy have $Z$ distributed according to SDSS observations (set ID $2 b$ ). In the third case, we assume that both galaxies and star clusters of all types follow a flat distribution in logarithmic bins (set ID 2c). The latter distribution serves to show how the merged $\mathrm{BH}$ population would change if the population of metal-poor galaxies inhabiting the volume scanned by the LVC contribute as much as metal-rich systems. Figure 9 shows how the $M_{\text {rem }}-a_{\text {rem }}$ plane would change as a consequence of such choices.

\footnotetext{
${ }^{14}$ Note that we refer to the metallicity of the stellar progenitor.
} 

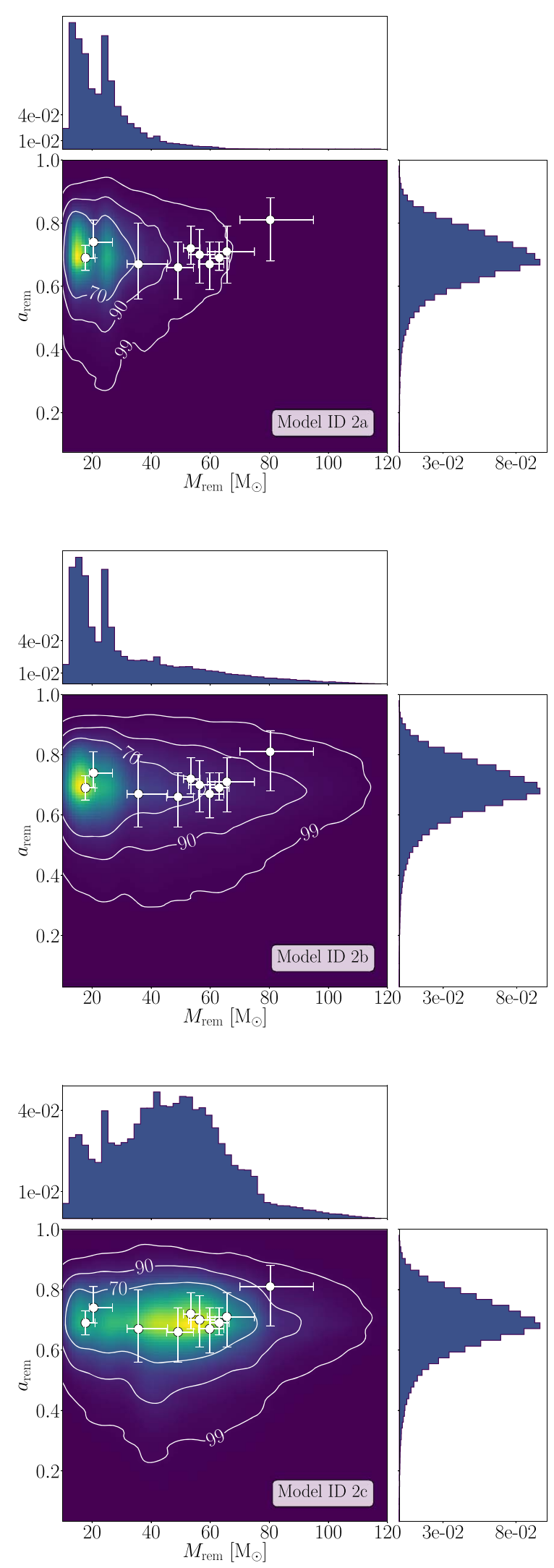

Figure 9. Remnant mass and spin plane in the assumption that the merger probability is independent of host metallicity. Top panel: galaxies and star clusters follow the observed $Z$ distribution, regardless of clusters' type (set ID 2a). Central panel: galaxies and open clusters follow the observed $Z$ distribution, while nuclear and globular clusters follow a logarithmically flat distribution (set ID 2b). Bottom panel: galaxies and star clusters follow a flat $Z$ distribution in logarithms, regardless of clusters' type (set ID2c).
As is apparent from Figure 9, assuming that environments' metallicity does not impact the merger probability, namely $f(Z)=1$, has strong implications for the $M_{\text {rem }}-a_{\text {rem }}$ plane. Indeed, it seems hard to reconcile the LVC detections with isolated and dynamical formation channels if we assume that both galaxies and star clusters have a $Z$ distribution shifted toward solar values as shown in SDSS observations. In this case, indeed, only the lightest BHs detected fall in the maximum of the $M_{\text {rem }}-a_{\text {rem }}$ plane, with the mass distribution peaking at $\sim 20-30 M_{\odot}$, as shown in the top panel of the figure. Assuming that star clusters have a flatter metallicity distribution, as in the fiducial model, but continuing to assume no metallicity-merger probability relation, leads to a slightly broader $M_{\text {rem }}$ distribution (central panel) that is still quite incompatible with observations. A way to obtain an $M_{\text {rem }}-a_{\text {rem }}$ distribution that embraces detected sources is to assume that the metallicity distribution of both galaxies and star clusters is flat in logarithms. For instance, by assuming a flat distribution in logarithmic metallicity values for both galaxies and star clusters, as shown in the bottom panel of Figure 9, we find that nine observed sources lie in the region containing half the total number of mock sources, thus implying a much better comparison with observations.

\subsection{Impact of the Formation Channel}

In this section, we discuss the impact of the formation channel in determining the $M_{\text {rem }}-a_{\text {rem }}$ plane. We assume either that all the mergers originate in the field $\left(f_{\text {iso }}=1\right.$, set ID 3 ) or in star clusters $\left(f_{\text {dyn }}=1\right.$, set ID 4a), leaving all the other parameters unchanged with respect to the fiducial model. As shown in Figure 10, the two channels produce significantly different patterns in the plane. This is due to two main factors: (i) the metallicity distribution, which is assumed to be different for galaxies and globular/nuclear clusters; (ii) the assumed correlation between observation probability and $\mathrm{BBH}$ primary mass.

The $M_{\text {rem }}$ distribution for isolated binaries shows three peaks at 15,25 , and $\sim 50 M_{\odot}$, and an abrupt decrease at values $\gtrsim 65 M_{\odot}$. Due to the sharp truncation at high mass, it seems quite hard to explain heavy remnants with an isolated origin, unless we assume that most observed BBHs formed several Gyr ago from metal-poor progenitors. Previous results exploring the cosmic evolution of merging BBHs pointed out that at least half the total mergers in the local universe formed from metal-poor progenitors at high redshift (Mapelli \& Giacobbo 2018; Mapelli et al. 2019). We stress that our work likely underestimates the contribution of BBHs that formed at high redshift and merged in the local universe, because our methodology cannot model the delay time self-consistently. Nonetheless, in our models, we can capture the contribution of high-redshift, metal-poor progenitors by modifying the metallicity distribution of galaxies. To this end, we explore three different possibilities, namely (i) that the distribution of galaxy metallicity equals that of local universe galaxies, as observed in the SDSS (model ID 3a); (ii) that the distribution of galaxy metallicity is shifted to values three times smaller than observed in the local universe (model ID $3 \mathrm{c}$ ) ${ }^{15}$ and iii) that the metallicity distribution is flat in logarithmic values (model

\footnotetext{
15 This corresponds to the case in which the metallicity distribution of BBH progenitors peaks at around $Z=0.001$, as discussed in Mapelli et al. (2019, see their Figure 4).
} 

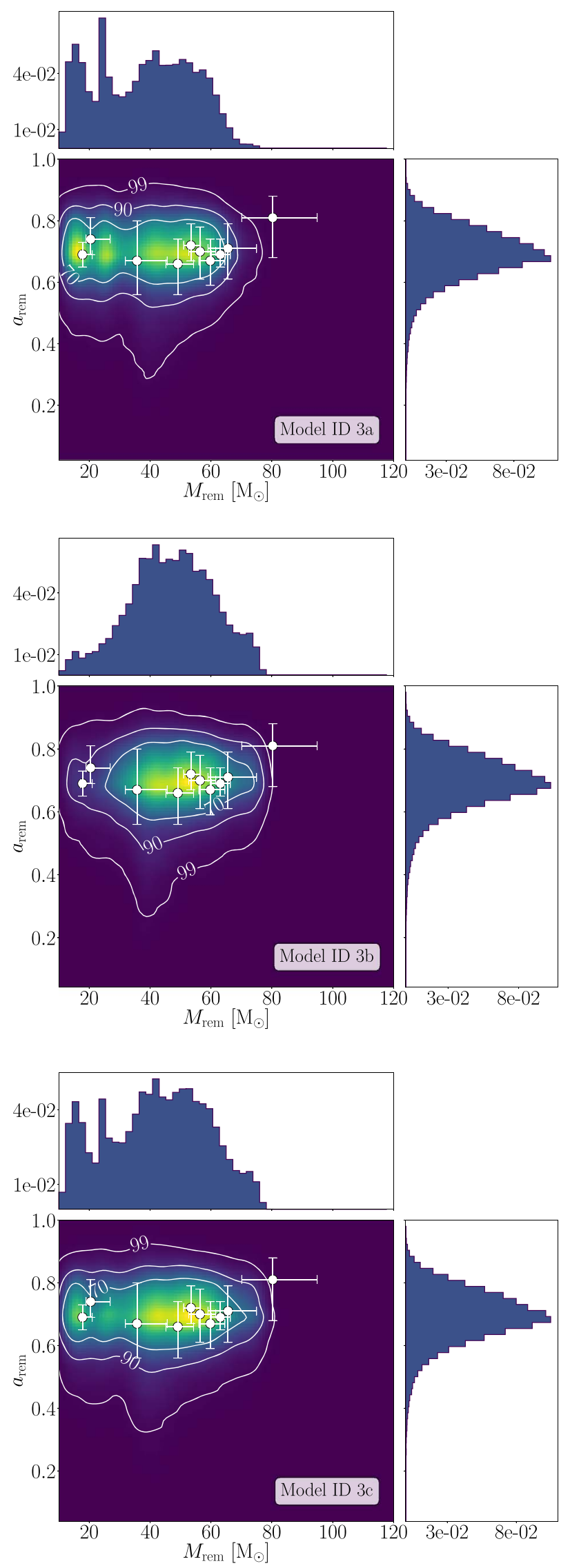

Figure 10. Remnant mass and spin distribution for models ID 3a (top panel), 3b (central panel), 3c (bottom panel).
ID 3b). In all three cases, we include the corrective function $f(Z)$ to the merger probability $P(Z)$. Figure 11 shows the remnant mass and spin distribution for all these models.

If all the observed sources have a dynamical origin, we find that 7 out of 10 detections fall in the maximum of the distribution. The $M_{\text {rem }}$ distribution in this case is very broad, with a single peak at $\sim 60 M_{\odot}$ and a tail extending up to $\lesssim 200 M_{\odot}$. A dynamical origin provides a suitable explanation for the heaviest merger product observed so far, GW170729, as it falls in a region encompassing $70 \%$ of all the modeled sources. The spin distribution is broader compared to isolated binaries, being characterized by an FWHM $\sim 0.2-0.25$ and a peak at 0.7 .

Clear differences among different channels and assumptions also emerge when comparing the distribution of binary masses $M_{\mathrm{BBH}}$ and mass ratios $q_{\mathrm{BBH}}$, as shown in the bottom row of panels in Figure 11. For comparison, we also show the binary combined mass-mass ratio distribution for binaries forming only in young clusters (set ID 4c).

Isolated binaries (left bottom panel) seem to preferentially form nearly equal-mass mergers, the mass ratio distribution being characterized by a nearly flat distribution in the $q_{\mathrm{BBH}} \simeq 0.2-0.7$ range, a steep rise at larger values, and a peak at $q_{\mathrm{BBH}}=0.9$ and $\mathrm{BBH}$ masses $M_{\mathrm{BBH}}=63 M_{\odot}$.

Dynamical binaries (central bottom panel) instead show a broad distribution in mass ratio values that embraces heavy detections. It must be noted that, in our model, such large distribution is obtained by construction, as we assume that the BBH mass ratio is randomly distributed between 1 and a minimum value $q_{\min }$. Also, we note that the mass distribution, compared to isolated binaries, extends to values larger than $100 M_{\odot}$. This is due to the combined effect of two assumptions: (i) isolated $\mathrm{BBH}$ masses are calculated via binary stellar evolution, whereas dynamical ones are calculated with single stellar evolution; and (ii) star clusters' metallicity distributions depend on their type, with young clusters having the same distribution as galaxies.

The latter panel in the bottom row of the figure shows dynamical BBHs formed only in young clusters. Recall that, in our models, this corresponds to the assumption that the metallicity distribution is the same as for galaxies, that there is no limit on the minimum mass ratio allowed, and that recycling depends only on the GW recoil kick after the previous merger. In this case, the BBH mass distribution broadens toward lower values compared to a more heterogeneous population of dynamical binaries, as shown in the central panel. This is due to the adopted metallicity distribution. This, combined with the looser assumption on the mass ratio, leads to a predominance of low-mass sources i.e., $M_{\mathrm{BBH}}<40 M_{\odot}$.

In our treatment, distinguishing between different dynamical environments (i.e., globular clusters, nuclear star clusters and young star clusters) corresponds to varying metallicity distribution, minimum mass ratio $q_{\min }$, and the multiple merger probability via $v_{\max }$. Figure 12 compares the $M_{\mathrm{rem}}-a_{\text {rem }}$ plane for BBHs forming either in young, globular, or nuclear clusters.

When comparing young and globular clusters, it is quite evident that the latter are characterized by a broader $M_{\text {rem }}$ distribution. This is due to the different assumption on the $Z$ distribution, which for young clusters is double-peaked at $Z \simeq 0.1 Z_{\odot}$ and $Z=Z_{\odot}$, while for nuclear and globular clusters it is equally distributed across logarithmic bins from 

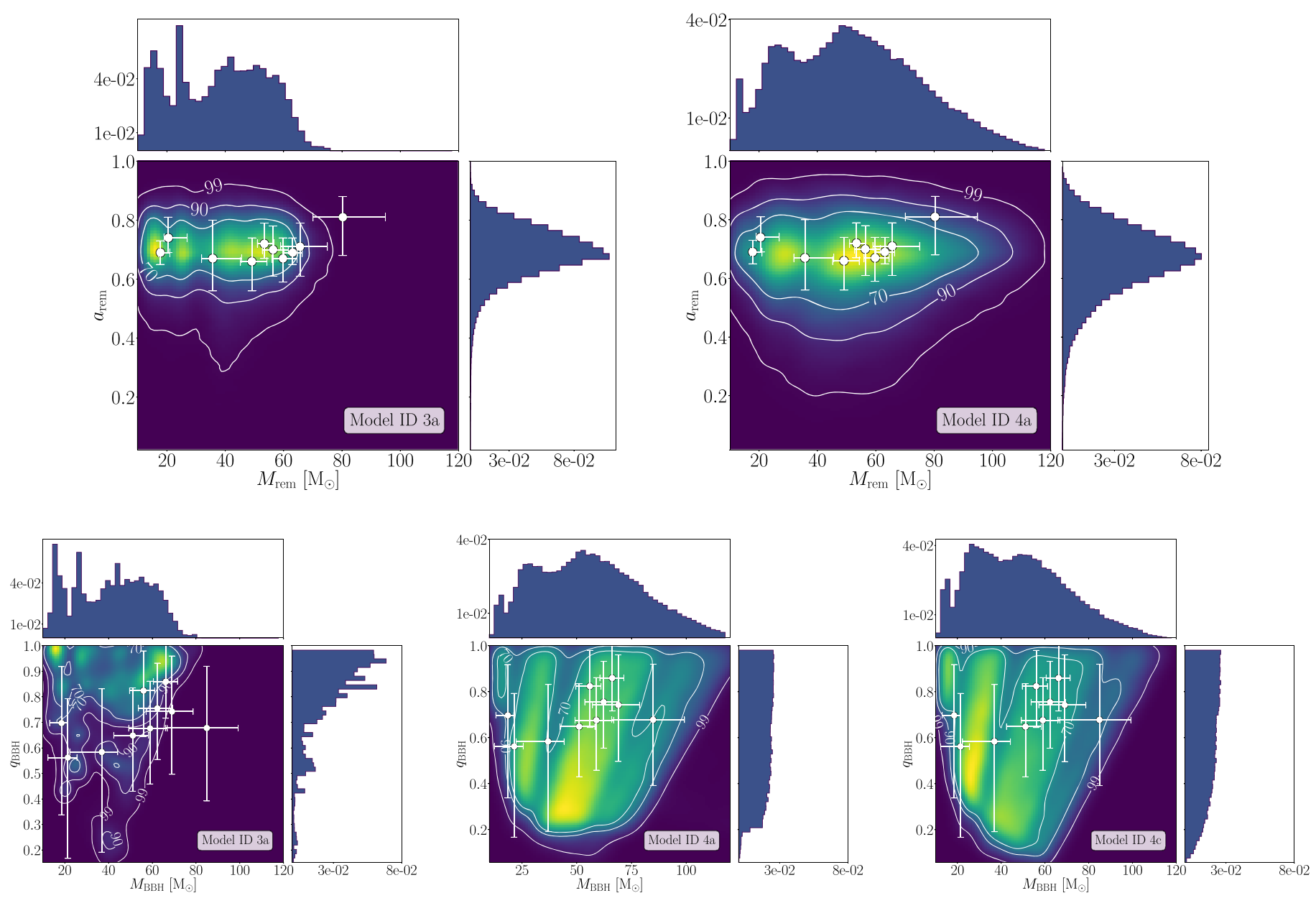

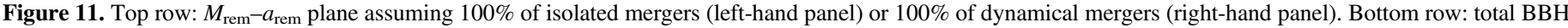

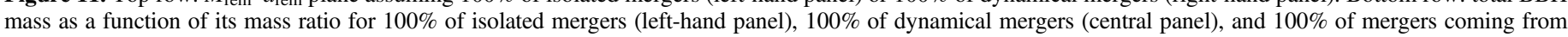
young clusters (right-hand panel).

$Z=0.01 Z_{\odot}$ up to solar values. In globular clusters, the higher escape velocity enables the formation of $\mathrm{BHs}$ with masses in the $120-200 M_{\odot}$ mass range, which are hard to explain under the assumptions made for young clusters. Hence, the potential detection of such massive $\mathrm{BHs}$ would allow us to place constraints on the metallicity distribution of the dynamical environments in which their progenitors developed.

Figure 12 also quantifies the importance of hierarchical $\mathrm{BH}$ mergers in the case of nuclear clusters. These are the dynamical environment in which multiple mergers are most likely to happen. In model ID $4 \mathrm{~d}$, we allow $\mathrm{BH}$ mergers to undergo a further merger depending on the GW recoil kick, while in model ID $4 \mathrm{e}$ we forbid recycling $\left(f_{\text {rec }}=0\right)$ for BHs in nuclear clusters. Repeated mergers are responsible for the long tail at the high end of the mass distribution, and they allow the formation of $\mathrm{BHs}$ as massive as $200 M_{\odot}$. In model $4 \mathrm{~d}$ (nuclear clusters with recycling), out of $10^{5}$ simulated BBHs, $\sim 5000 \mathrm{BHs}$ undergo two mergers, 115 undergo three mergers, and four undergo four mergers. BHs undergoing two or three subsequent mergers can reach masses up to $250 M_{\odot}$. Finding a number of BHs with such large masses would provide crucial insights regarding a) the probability of multiple mergers, and b) the merger rate from dense and massive clusters compared to other formation channels. We note that this result does not account for the possible formation of massive black holes through (multiple) mergers of massive stars (Portegies Zwart et al. 2004; Mapelli 2016). This alternative channel might lead to the formation of BBHs with mass $>>100 M_{\odot}$ even in dense young star clusters, as described in di Carlo et al. (2019a, 2019b).

A further model worthy of investigation relies upon the assumption that $\mathrm{BBH}$ mergers are equally contributed by isolated and dynamical binaries $\left(f_{\text {iso }}=f_{\text {dyn }}=0.5\right)$ and that all cluster types contribute equally to dynamical mergers $\left(f_{\mathrm{GC}}=f_{\mathrm{NC}}=f_{\mathrm{YC}}\right.$, set ID 5). The $M_{\mathrm{rem}}-a_{\mathrm{rem}}$ and $q_{\mathrm{BBH}}-M_{\mathrm{BBH}}$ planes corresponding to such a model are shown in Figure 13. Having an equal contribution from dynamical and isolated binaries widens the $\mathrm{BBH}$ mass ratio distribution. Since $q_{\mathrm{BBH}}$ distribution is narrow and peaked around unity for isolated binaries, whereas it is flat for our dynamical ones by construction, increasing the percentage of dynamical binaries leads to a larger amount of unequal mass binaries, thus increasing the match between observations and models. At the same time, a larger number of dynamical mergers reduces the number of light remnant BHs, $M_{\text {rem }} \simeq 20-40 M_{\odot}$, making it harder to match observations and models in the $M_{\text {rem }}-a_{\text {rem }}$ plane.

\subsection{Impact of the Maximum BH Mass}

One of the main open questions about $\mathrm{BH}$ formation is the maximum mass $\left(M_{\mathrm{BH} \text { max }}\right)$ of a $\mathrm{BH}$ born from a single star with zero-age main-sequence mass $m_{\text {ZAMS }} \leqslant 230 M_{\odot}$. This is strongly affected by (pulsational) pair instability 

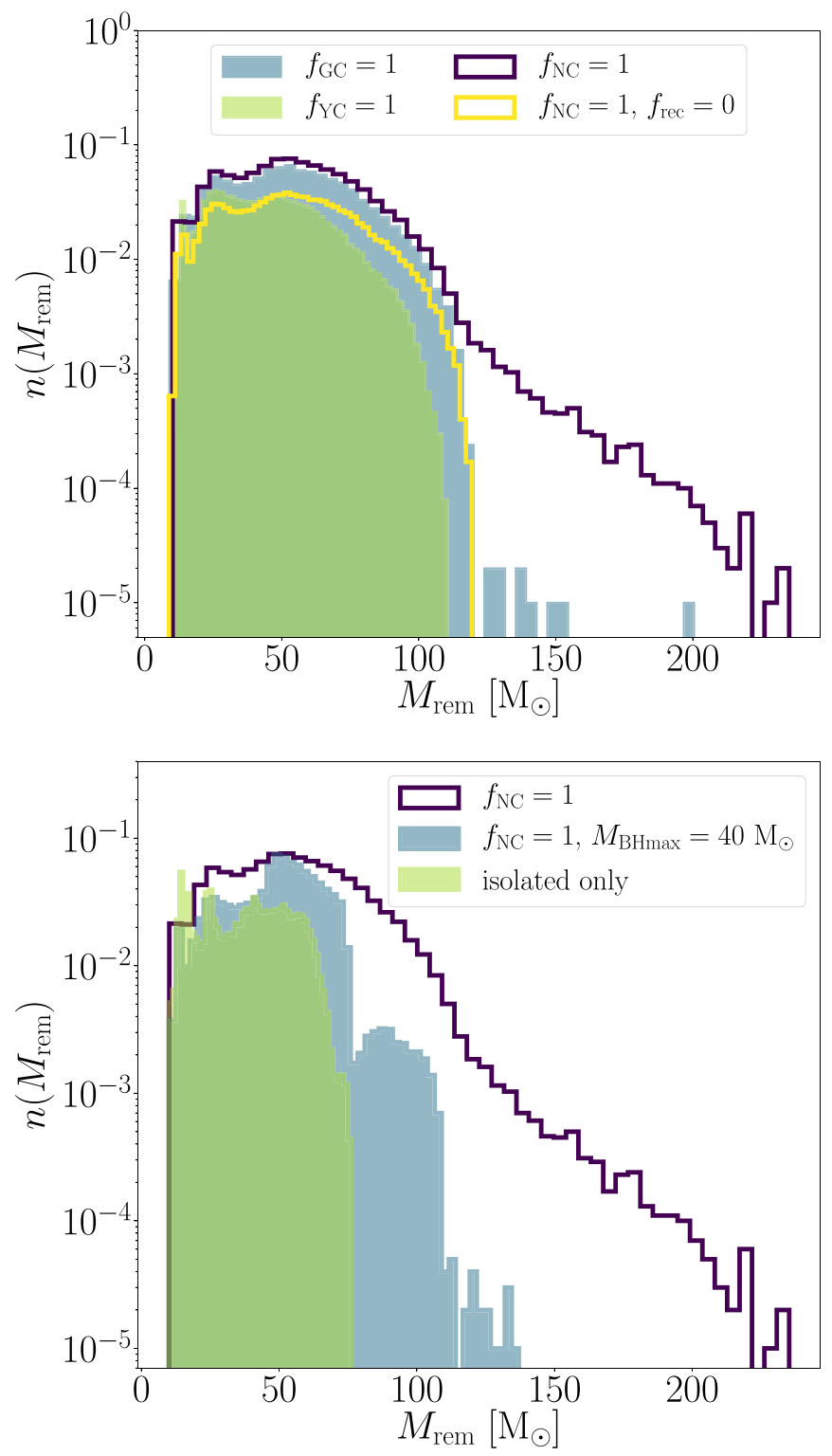

Figure 12. Top panel: $M_{\text {rem }}$ distribution for globular (blue steps, model $4 \mathrm{~b}$ ), young (green steps, model 4c), and nuclear clusters (purple steps, model 4d) even in the case with no recycling allowed (yellow steps, model 4e). Bottom panel: $M_{\mathrm{rem}}$ distribution for nuclear clusters assuming MOBSE BH mass spectrum (model $4 \mathrm{~d}$ ) and limiting this mass spectrum to a maximum value $M_{\mathrm{BH} \max }=40 M_{\odot}(\operatorname{model} 4 \mathrm{~d} \dagger)$.

(Belczynski et al. 2016a; Spera \& Mapelli 2017; Woosley 2017, 2019; Giacobbo et al. 2018; Marchant et al. 2019; Stevenson et al. 2019; Renzo et al. 2020), by stellar rotation (Mapelli et al. 2020), by uncertainty on nuclear reaction rates (Farmer et al. 2019), and by the collapse of a residual hydrogen envelope (Mapelli et al. 2020). As a result, $M_{\mathrm{BH} \max }$ might be as low as $\sim 40-45 M_{\odot}$ (Belczynski et al. 2016b) or as high as $\sim 65$ $M_{\odot}$ (Giacobbo et al. 2018).

To explore the role of $M_{\mathrm{BHmax}}$ in shaping the remnant mass distribution, we run a further model, named $4 \mathrm{~d} \dagger$, which assumes $M_{\mathrm{BHmax}}=40 M_{\odot}$ in our MOBSE models. The bottom panel of Figure 12 shows the comparison between models $4 \mathrm{~d}$ (single $\mathrm{BH}$ masses from $\mathrm{MOBSE}$ ) and $4 \mathrm{~d} \dagger$ (single $\mathrm{BH}$ masses capped at $40 M_{\odot}$ ). As shown in the plot, limiting the maximum mass of zeroth-generation BHs leads to a sharp drop of the
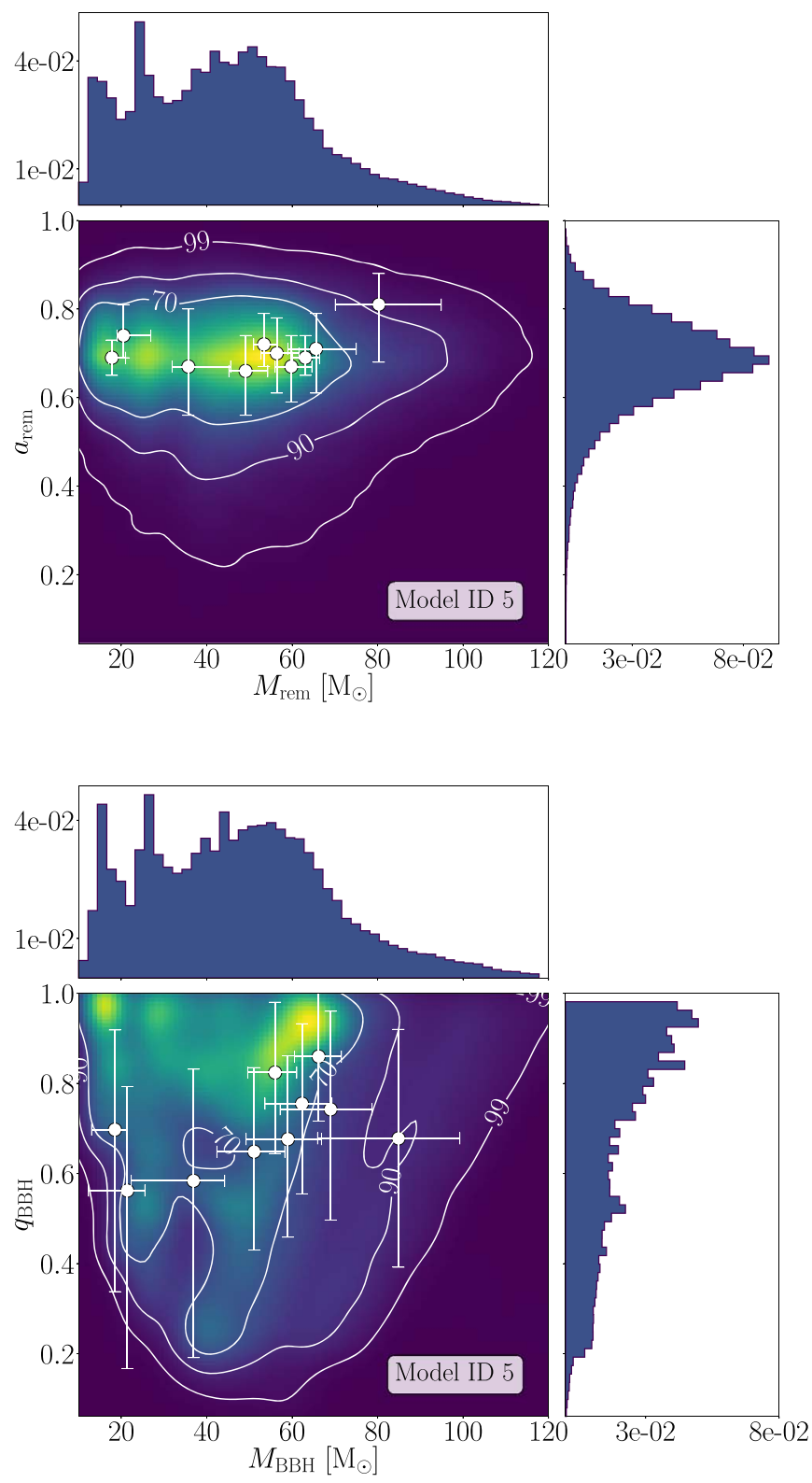

Figure 13. Top panel: $M_{\mathrm{rem}}-a_{\mathrm{rem}}$ plane assuming that BBH mergers are equally distributed among isolated and dynamical channels $\left(f_{\text {iso }}=f_{\text {dyn }}\right)$, and among different cluster types $\left(f_{\mathrm{YC}}=f_{\mathrm{GC}}=f_{\mathrm{NC}}\right)$, i.e., set ID 5. Bottom panel: same as above, but here the BBH mass-mass ratio plane is shown.

number of systems with $M_{\text {rem }} \geqslant 120-130 M_{\odot}$. The distribution of model $4 \mathrm{~d} \dagger$ with $f_{\mathrm{NC}}=1$ shows two peaks: one at $\sim 50 M_{\odot}$ and the other at $\sim 100 M_{\odot}$.

\subsection{Caveats}

In this work, we developed a self-consistent statistical approach to construct catalogs of $\mathrm{BBH}$ mergers forming via different channels and in various environments. Our tool allowed us to explore a wide portion of the phase space and to place constraints on the role played by different parameters in determining the properties of $\mathrm{BBH}$ merger populations. Although quite fast and based on a set of physically motivated assumptions, our method comes with a number of caveats, which we discuss below. Overcoming these limitations requires a more in-depth study that faces the computational challenge of 
simultaneously modeling star clusters and isolated binary populations together with their host galaxy, while taking into account the chemo-dynamical evolution of the whole system. Our tool can serve as a basis to understand which parameters are the most effective in influencing the properties of $\mathrm{BBH}$ populations, and can be used to compare with observations but also to understand what kind of more detailed follow-up models are needed to improve our knowledge of $\mathrm{BBH}$ physics and evolution.

Despite its versatility and wide applicability to study both dynamical and isolated binaries, our tool does not include any treatment for stellar collisions, which might affect the BH mass spectrum. We excluded this feature because it is currently rather poorly understood, since the number of studies addressing this aspect of dynamics is rather low (see, e.g., di Carlo et al. 2019a). The tool does not take into account the metallicity gradient in modeling BBH galaxy hosts, although this issue is partly addressed via defining different metallicity distribution choices (see, for instance, models ID 2a, b, and c). Our analysis excludes $\mathrm{BBH}$ mergers forming via alternative processes, like $\mathrm{BBH}$ formation around an SMBH or in an AGN disk (McKernan et al. 2012, 2014, 2018; Bartos et al. 2017; Yang et al. 2019), or in triples (Antonini \& Perets 2012; Fragione et al. 2019; Hoang et al. 2018; Arca Sedda 2020). Moreover, our tool does not account for chemically homogeneous binary evolution (Marchant et al. 2016). This formation channel can produce remnants with mass $>130 M_{\odot}$, much higher than the limit posed by other models for isolated $\mathrm{BBH}$ formation. The fraction of chemically homogeneous stars over the total population might be larger at low metallicity (Ramachandran et al. 2019), but the delay time for BBHs from chemically homogeneous stars is short $(<0.4$ Gyr Marchant et al. 2016), thus limiting the possibility that this type of mergers take place at $z<1$.

In our method, we do not explicitly consider delay times in creating our mock BBHs. Because BBHs forming in metalpoor galaxies at high redshift can constitute almost half of the mergers taking place at redshift zero (Mapelli et al. 2019), we encoded the information about the delay time in the choice of metallicity distribution as discussed in Section 4.1. Regarding dynamical binaries, we do not make any assumption on the possible relation between the delay time and the $\mathrm{BBH}$ total mass and spin in star clusters. In a future versions of our tool, we will properly incorporate the time delay to self-consistently quantify any possible correlation between this quantity and $\mathrm{BBH}$ population properties.

Finally, we do not account for different stellar evolution recipes or different spin distributions. The role played by these ingredients has already been discussed in our previous paper (Arca Sedda \& Benacquista 2019).

\section{Conclusions}

We use a statistical approach to build BBH samples to be compared with observations. Our model takes into account the effects arising from $\mathrm{BBH}$ birth places, formation channels, initial metallicity, progenitor's natal mass, kicks, and spins. Our results can be summarized as follows.

1. We presented a versatile and self-consistent approach to construct catalogs of $\mathrm{BBH}$ mergers forming through different channels, which allows a fast exploration of a wide parameter space (Figure 1). Our approach is an effective alternative to the computational challenge of dynamical simulations, but contains several approximations (e.g., the mass function of BBHs in star clusters and the time delays).

2. We show that the metallicity distribution of parent galaxies is a crucial parameter to assess the distribution of $\mathrm{BBH}$ masses, and can severely affect theoretical models. Moreover, we discuss the impact of post-merger GW recoil, which can limit the probability for a merged $\mathrm{BH}$ to undergo another merger event (Figures 2 and 3).

3. Using our technique, we create samples of $100,000 \mathrm{BBH}$ mergers forming either in isolation or via dynamical interactions in star clusters. For each merger, we calculate the remnant mass and spin and show how the global distribution compares with $\mathrm{O} 1+\mathrm{O} 2$ LVC BBHs. The results for our fiducial model are shown in Figure 4.

4. By comparing simulated and observed BBHs, we show that the fiducial model matches fairly well the properties of mergers observed during $\mathrm{O} 1$ and $\mathrm{O} 2$ (Figure 6).

5. Based on the assumptions of our model, if the number of sources with remnant mass $\geqslant 70 M_{\odot}$ is significantly larger than that of sources with remnant mass $\leqslant 30 M_{\odot}$, dynamical BBHs dominate the population, while the absence of sources with $\geqslant 70 M_{\odot}$ implies that the isolated channel contributes to most of the $\mathrm{BBH}$ population in the local universe (Table 2).

6. According to our fiducial model, we predict that at least one $\mathrm{BBH}$ out of 100 will leave a remnant with a mass $90<M_{\mathrm{rem}} / M_{\odot}<110$, and one out of 1000 will have $110<M_{\mathrm{rem}} / M_{\odot}<250 M_{\odot}$, i.e., in the range of intermediate-mass black holes (Figure 7).

7. We investigate a possible formation route for the heaviest BBH reported to date, GW170729 (Abbott et al. 2019b), as the product of a series of mergers taking place in a dense star cluster. We find that the observed mass and spin of GW170729 can be explained by 1-3 subsequent mergers occurring in a dense cluster with velocity dispersion $\mathrm{O}\left(100 \mathrm{~km} \mathrm{~s}^{-1}\right)$ and metallicity in the range $Z=0.0002-0.002$.

8. We explore how different parameters affect the results. We demonstrate that the metallicity distribution and the relative amount of dynamical and isolated channels are the most important parameters determining the remnant mass distribution. Our results suggest that both the dynamical and isolated channel contribute to the overall population of $\mathrm{BBH}$ mergers.

The authors are grateful to the referee for comments and suggestions that helped to improve an earlier version of this manuscript. M.A.S. acknowledges financial support from the Alexander von Humboldt Foundation and the Federal Ministry for Education and Research for the research project "The evolution of black holes from stellar to galactic scales.” M.M. acknowledges financial support by the European Research Council for the ERC Consolidator grant DEMOBLACK, under contract no. 770017. M.S. acknowledges funding from the European Union's Horizon 2020 research and innovation programme under the MarieSklodowska-Curie grant agreement No. 794393. M.B. acknowledges support provided while serving at the National Science Foundation through award 1755085. Any opinions, findings, conclusions, or recommendations expressed in this material are those of the authors and do not necessarily reflect the views of the 
National Science Foundation. This work benefited from support by the International Space Science Institute (ISSI), Bern, Switzerland, through its International Team programme ref. no. 393 "The Evolution of Rich Stellar Populations \& BH Binaries (2017-18)," by the Sonderforschungsbereich SFB 881 "The Milky Way System" of the German Research Foundation (DFG), and by the COST Action CA16104 "GWverse."

\section{ORCID iDs}

Manuel Arca Sedda (i) https://orcid.org/0000-0002-3987-0519 Michela Mapelli (iD https://orcid.org/0000-0001-8799-2548 Mario Spera (1) https://orcid.org/0000-0003-0930-6930 Nicola Giacobbo (1) https://orcid.org/0000-0002-8339-0889

\section{References}

Abbott, B. P., Abbott, R., Abbott, T. D., et al. 2016a, PhRvD, 93, 122003 Abbott, B. P., Abbott, R., Abbott, T. D., et al. 2016b, PhRvL, 116, 061102 Abbott, B. P., Abbott, R., Abbott, T. D., et al. 2016c, PhRvL, 116, 241103 Abbott, B. P., Abbott, R., Abbott, T. D., et al. 2017a, PhRvL, 118, 221101 Abbott, B. P., Abbott, R., Abbott, T. D., et al. 2017b, PhRvL, 119, 141101 Abbott, B. P., Abbott, R., Abbott, T. D., et al. 2017c, PhRvL, 119, 161101 Abbott, B. P., Abbott, R., Abbott, T. D., et al. 2017d, ApJL, 848, L13 Abbott, B. P., Abbott, R., Abbott, T. D., et al. 2019a, PhRvX, 9, 031040 Abbott, B. P., Abbott, R., Abbott, T. D., et al. 2019b, ApJL, 882, L24 Amaro-Seoane, P., \& Chen, X. 2016, MNRAS, 458, 3075 Antonini, F., Chatterjee, S., Rodriguez, C. L., et al. 2016, ApJ, 816, 65 Antonini, F., Gieles, M., \& Gualandris, A. 2019, MNRAS, 486, 5008 Antonini, F., \& Perets, H. B. 2012, ApJ, 757, 27

Antonini, F., \& Rasio, F. A. 2016, ApJ, 831, 187

Antonini, F., Toonen, S., \& Hamers, A. S. 2017, ApJ, 841, 77 Arca Sedda, M. 2020, ApJ, 891, 47

Arca Sedda, M., \& Benacquista, M. 2019, MNRAS, 482, 2991 Arca Sedda, M., \& Capuzzo-Dolcetta, R. 2019, MNRAS, 483, 152 Arca Sedda, M., \& Gualandris, A. 2018, MNRAS, 477, 4423 Arca Sedda, M., Li, G., \& Kocsis, B. 2018, arXiv:1805.06458 Askar, A., Szkudlarek, M., Gondek-Rosińska, D., Giersz, M., \& Bulik, T. 2017, MNRAS, 464, L36

Baker, J. G., Centrella, J., Choi, D.-I., Koppitz, M., \& van Meter, J. 2006, PhRvL, 96, 111102

Banerjee, S. 2017, MNRAS, 467, 524

Banerjee, S. 2018, MNRAS, 473, 909

Banerjee, S., Baumgardt, H., \& Kroupa, P. 2010, MNRAS, 402, 371

Barrett, J. W., Gaebel, S. M., Neijssel, C. J., et al. 2018, MNRAS, 477, 4685

Bartos, I., Kocsis, B., Haiman, Z., \& Márka, S. 2017, ApJ, 835, 165

Bavera, S. S., Fragos, T., Qin, Y., et al. 2020, A\&A, 635, A97

Belczynski, K., Bulik, T., Fryer, C. L., et al. 2010, ApJ, 714, 1217

Belczynski, K., Heger, A., Gladysz, W., et al. 2016a, A\&A, 594, A97

Belczynski, K., Holz, D. E., Bulik, T., \& O'Shaughnessy, R. 2016b, Natur, 534,512

Belczynski, K., Kalogera, V., \& Bulik, T. 2002, ApJ, 572, 407

Belczynski, K., Klencki, J., Meynet, G., et al. 2020, A\&A, 636, 104

Bird, S., Cholis, I., Muñoz, J. B., et al. 2016, PhRvL, 116, 201301

Bouffanais, Y., Mapelli, M., Gerosa, D., et al. 2019, ApJ, 886, 25

Broadhurst, T., Diego, J. M., \& Smoot, G. I. 2018, arXiv:1802.05273

Campanelli, M., Lousto, C. O., Marronetti, P., \& Zlochower, Y. 2006, PhRvL, 96,111101

Campanelli, M., Lousto, C. O., Zlochower, Y., \& Merritt, D. 2007, PhRvL, 98, 231102

Capano, C., Harry, I., Privitera, S., \& Buonanno, A. 2016, PhRvD, 93, 124007

Casares, J., Jonker, P. G., \& Israelian, G. 2017, in Handbook of Supernovae, ed. A. W. Alsabti \& P. Murdin (Berlin: Springer), 1499

di Carlo, U. N., Giacobbo, N., Mapelli, M., et al. 2019a, MNRAS, 487, 2947 di Carlo, U. N., Mapelli, M., Bouffanais, Y., et al. 2019b, arXiv:1911.01434 di Carlo, U. N., Mapelli, M., Giacobbo, N., et al. 2020, arXiv:2004.09525

Do, T., Kerzendorf, W., Winsor, N., et al. 2015, ApJ, 809, 143

Doctor, Z., Wysocki, D., O'Shaughnessy, R., Holz, D. E., \& Farr, B. 2020, ApJ, 893, 35

Dominik, M., Belczynski, K., Fryer, C., et al. 2013, ApJ, 779, 72

Dominik, M., Berti, E., O'Shaughnessy, R., et al. 2015, ApJ, 806, 263

Downing, J. M. B., Benacquista, M. J., Giersz, M., \& Spurzem, R. 2010, MNRAS, 407, 1946
Eldridge, J. J., Stanway, E. R., Xiao, L., et al. 2017, PASA, 34, e058

Farmer, R., Renzo, M., de Mink, S. E., Marchant, P., \& Justham, S. 2019, ApJ, 887,53

Farr, W. M., Stevenson, S., Miller, M. C., et al. 2017, Natur, 548, 426

Fernández, J. J., \& Kobayashi, S. 2019, MNRAS, 487, 1200

Fishbach, M., \& Holz, D. E. 2017, ApJL, 851, L25

Fishbach, M., Holz, D. E., \& Farr, B. 2017, ApJL, 840, L24

Fragione, G., Grishin, E., Leigh, N. W. C., Perets, H. B., \& Perna, R. 2019, MNRAS, 488,47

Gallazzi, A., Charlot, S., Brinchmann, J., White, S. D. M., \& Tremonti, C. A. 2005, MNRAS, 362, 41

Georgiev, I. Y., Hilker, M., Puzia, T. H., Goudfrooij, P., \& Baumgardt, H. 2009, MNRAS, 396, 1075

Gerosa, D., \& Berti, E. 2017, PhRvD, 95, 124046

Gerosa, D., Berti, E., O'Shaughnessy, R., et al. 2018, PhRvD, 98, 084036

Giacobbo, N., \& Mapelli, M. 2020, ApJ, 891, 141

Giacobbo, N., Mapelli, M., \& Spera, M. 2018, MNRAS, 474, 2959

González, J. A., Sperhake, U., Brügmann, B., Hannam, M., \& Husa, S. 2007, PhRvL, 98, 091101

Harris, W. E., Morningstar, W., Gnedin, O. Y., et al. 2014, ApJ, 797, 128

Hoang, B.-M., Naoz, S., Kocsis, B., Farr, W., \& McIver, J. 2019, ApJL, 875,31

Hoang, B.-M., Naoz, S., Kocsis, B., Rasio, F. A., \& Dosopoulou, F. 2018, ApJ, 856,140

Hofmann, F., Barausse, E., \& Rezzolla, L. 2016, ApJL, 825, L19

Hong, J., Vesperini, E., Askar, A., et al. 2018, MNRAS, 480, 5645

Hughes, S. A., \& Blandford, R. D. 2003, ApJL, 585, L101

Hurley, J. R., Tout, C. A., \& Pols, O. R. 2002, MNRAS, 329, 897

Jiménez-Forteza, X., Keitel, D., Husa, S., et al. 2017, PhRvD, 95, 064024

Kimball, C., Berry, C. P. L., \& Kalogera, V. 2020, RNAAS, 4, 2

Kroupa, P. 2001, MNRAS, 322, 231

Kruckow, M. U., Tauris, T. M., Langer, N., Kramer, M., \& Izzard, R. G. 2018, MNRAS, 481, 1908

Kumamoto, J., Fujii, M. S., \& Tanikawa, A. 2019, MNRAS, 486, 3942

Lamers, H. J. G. L. M., Kruijssen, J. M. D., Bastian, N., et al. 2017, A\&A, 606, A85

Lee, H. M. 1995, MNRAS, 272, 605

Lousto, C. O., \& Zlochower, Y. 2008, PhRvD, 77, 044028

Lousto, C. O., Zlochower, Y., Dotti, M., \& Volonteri, M. 2012, PhRvD, 85, 084015

Mapelli, M. 2016, MNRAS, 459, 3432

Mapelli, M., \& Bressan, A. 2013, MNRAS, 430, 3120

Mapelli, M., Colpi, M., \& Zampieri, L. 2009, MNRAS, 395, L71

Mapelli, M., \& Giacobbo, N. 2018, MNRAS, 479, 4391

Mapelli, M., Giacobbo, N., Ripamonti, E., \& Spera, M. 2017, MNRAS, 472, 2422

Mapelli, M., Giacobbo, N., Santoliquido, F., \& Artale, M. C. 2019, MNRAS, 487, 2

Mapelli, M., Huwyler, C., Mayer, L., Jetzer, P., \& Vecchio, A. 2010, ApJ, 719, 987

Mapelli, M., Spera, M., Montanari, E., et al. 2020, ApJ, 888, 76

Marchant, P., Langer, N., Podsiadlowski, P., Tauris, T. M., \& Moriya, T. J. 2016, A\&A, 588, A50

Marchant, P., Renzo, M., Farmer, R., et al. 2019, ApJ, 882, 36

Martynov, D. V., Hall, E. D., Abbott, B. P., et al. 2016, PhRvD, 93, 112004

McKernan, B., Ford, K. E. S., Bellovary, J., et al. 2018, ApJ, 866, 66

McKernan, B., Ford, K. E. S., Kocsis, B., Lyra, W., \& Winter, L. M. 2014, MNRAS, 441, 900

McKernan, B., Ford, K. E. S., Lyra, W., \& Perets, H. B. 2012, MNRAS, 425,460

Miller, M. C., \& Hamilton, D. P. 2002, ApJ, 576, 894

Miller, M. C., \& Lauburg, V. M. 2009, ApJ, 692, 917

Morawski, J., Giersz, M., Askar, A., \& Belczynski, K. 2018, MNRAS, 481,2168

Neijssel, C. J., Vigna-Gómez, A., Stevenson, S., et al. 2019, MNRAS, 490, 3740

Netopil, M., Paunzen, E., Heiter, U., \& Soubiran, C. 2016, A\&A, 585, A150 Neumayer, N., Seth, A., \& Boeker, T. 2020, arXiv:2001.03626

O'Leary, R. M., Kocsis, B., \& Loeb, A. 2009, MNRAS, 395, 2127

O'Leary, R. M., Meiron, Y., \& Kocsis, B. 2016, ApJL, 824, L12

Paudel, S., Lisker, T., \& Kuntschner, H. 2011, MNRAS, 413, 1764

Perna, R., Wang, Y.-H., Farr, W. M., Leigh, N., \& Cantiello, M. 2019, ApJL, 878, L1

Pilyugin, L. S., Grebel, E. K., \& Kniazev, A. Y. 2014, AJ, 147, 131

Portegies Zwart, S. F., Baumgardt, H., Hut, P., Makino, J., \& McMillan, S. L. W. 2004, Natur, 428, 724 
Portegies Zwart, S. F., \& McMillan, S. L. W. 2000, ApJL, 528, L17

Portegies Zwart, S. F., McMillan, S. L. W., \& Gieles, M. 2010, ARA\&A, 48, 431

Pretorius, F. 2005, CQGra, 22, 425

Qin, Y., Fragos, T., Meynet, G., et al. 2018, A\&A, 616, A28

Ramachandran, V., Hamann, W. R., Oskinova, L. M., et al. 2019, A\&A, 625, A104

Rasskazov, A., \& Kocsis, B. 2019, ApJ, 881, 20

Rastello, S., Amaro-Seoane, P., Arca-Sedda, M., et al. 2019, MNRAS, 483, 1233

Remillard, R. A., \& McClintock, J. E. 2006, ARA\&A, 44, 49

Renzo, M., Farmer, R., Justham, S., et al. 2020, arXiv:2002.05077

Rezzolla, L., Barausse, E., Dorband, E. N., et al. 2008, PhRvD, 78, 044002

Rodriguez, C. L., Amaro-Seoane, P., Chatterjee, S., \& Rasio, F. A. 2018, PhRvL, 120, 151101

Rodriguez, C. L., Chatterjee, S., \& Rasio, F. A. 2016, PhRvD, 93, 084029

Rodriguez, C. L., \& Loeb, A. 2018, ApJL, 866, L5

Rodriguez, C. L., Morscher, M., Pattabiraman, B., et al. 2015, PhRvL, 115, 051101

Rodriguez, C. L., Zevin, M., Amaro-Seoane, P., et al. 2019, PhRvD, 100, 043027

Rossa, J., van der Marel, R. P., Böker, T., et al. 2006, AJ, 132, 1074

Samsing, J. 2018, PhRvD, 97, 103014
Sana, H., de Mink, S. E., de Koter, A., et al. 2012, Sci, 337, 444

Sigurdsson, S., \& Phinney, E. S. 1993, ApJ, 415, 631

Spera, M., \& Mapelli, M. 2017, MNRAS, 470, 4739

Spera, M., Mapelli, M., \& Bressan, A. 2015, MNRAS, 451, 4086

Spera, M., Mapelli, M., Giacobbo, N., et al. 2019, MNRAS, 485, 889

Sperhake, U. 2015, CQGra, 32, 124011

Stephan, A. P., Naoz, S., Ghez, A. M., et al. 2016, MNRAS, 460, 3494

Stevenson, S., Berry, C. P. L., \& Mandel, I. 2017, MNRAS, 471, 2801

Stevenson, S., Sampson, M., Powell, J., et al. 2019, ApJ, 882, 121

Talbot, C., \& Thrane, E. 2017, PhRvD, 96, 023012

Tutukov, A., \& Yungelson, L. 1973, NInfo, 27, 70

VanLandingham, J. H., Miller, M. C., Hamilton, D. P., \& Richardson, D. C. 2016, ApJ, 828, 77

Wang, L., Spurzem, R., Aarseth, S., et al. 2016, MNRAS, 458, 1450

Wen, L. 2003, ApJ, 598, 419

Woosley, S. E. 2017, ApJ, 836, 244

Woosley, S. E. 2019, ApJ, 878, 49

Yang, Y., Bartos, I., Haiman, Z., et al. 2019, ApJ, 876, 122

Zevin, M., Pankow, C., Rodriguez, C. L., et al. 2017, ApJ, 846, 82

Zevin, M., Samsing, J., Rodriguez, C., Haster, C.-J., \& Ramirez-Ruiz, E. 2019, ApJ, 871, 91

Ziosi, B. M., Mapelli, M., Branchesi, M., \& Tormen, G. 2014, MNRAS, 441,3703 